Article

\title{
A Novel Approach to Generalized Intuitionistic Fuzzy Soft Sets and Its Application in Decision Support System
}

\author{
Muhammad Jabir Khan ${ }^{1}\left(\mathbb{D}\right.$, Poom Kumam ${ }^{1,2,3, * \mathbb{C}}$, Peide Liu ${ }^{4}$ and Wiyada Kumam ${ }^{5, *(\mathbb{D})}$ \\ and Shahzaib Ashraf ${ }^{6}$ \\ 1 KMUTTFixed Point Research Laboratory, SCL 802 Fixed Point Laboratory \& Department of Mathematics, \\ Faculty of Science, King Mongkut's University of Technology Thonburi (KMUTT), 126 Pracha-Uthit Road, \\ Bang Mod, Thrung Khru, Bangkok 10140, Thailand \\ 2 Center of Excellence in Theoretical and Computational Science (TaCS-CoE), Science Laboratory Building, \\ Faculty of Science, King Mongkut's University of Technology Thonburi (KMUTT), 126 Pracha-Uthit Road, \\ Bang Mod, Thrung Khru, Bangkok 10140, Thailand \\ 3 Department of Medical Research, China Medical University Hospital, China Medical University, \\ Taichung 40402, Taiwan \\ 4 School of Management Science and Engineering, Shandong University of Finance and Economics, \\ Jinan 250014, China \\ 5 Program in Applied Statistics, Department of Mathematics and Computer Science, Faculty of Science and \\ Technology, Rajamangala University of Technology Thanyaburi (RMUTT), Thanyaburi, \\ Pathumthani 12110, Thailand \\ 6 Department of Mathematics, Abdul wali Khan University, Mardan 23200, Pakistan \\ * Correspondence: poom.kum@kmutt.ac.th (P.K.); wiyada.kum@rmutt.ac.th (W.K.)
}

Received: 24 June 2019; Accepted: 28 July 2019; Published: 13 August 2019

\begin{abstract}
The basic idea underneath the generalized intuitionistic fuzzy soft set is very constructive in decision making, since it considers how to exploit an extra intuitionistic fuzzy input from the director to make up for any distortion in the information provided by the evaluation experts, which is redefined and clarified by F. Feng. In this paper, we introduced a method to solve decision making problems using an adjustable weighted soft discernibility matrix in a generalized intuitionistic fuzzy soft set. We define the threshold functions like mid-threshold, top-bottom-threshold, bottom-bottom-threshold, top-top-threshold, med-threshold function and their level soft sets of the generalized intuitionistic fuzzy soft set. After, we proposed two algorithms based on threshold functions, a weighted soft discernibility matrix and a generalized intuitionistic fuzzy soft set and also to show the supremacy of the given methods we illustrate a descriptive example using a weighted soft discernibility matrix in the generalized intuitionistic fuzzy soft set. Results indicate that the proposed method is more effective and generalized over all existing methods of the fuzzy soft set.
\end{abstract}

Keywords: generalized intuitionistic fuzzy soft set; adjustable approach; soft discernibility matrix; multi-criteria decision making

\section{Introduction}

The real world is full of imprecision, vagueness and uncertainty. In our daily life, we deal mostly with unclear concepts rather than exact ones. Dealing with imprecision is a big problem in many areas 
such as economics, medical science, social science, environmental science and engineering. In recent years, model vagueness has become interested in many authors. Many classical theories such as fuzzy set theory [1], probability theory, vague set theory [2], rough set theory [3], intuitionistic fuzzy set [4] and interval mathematics [5] are well known and effectively model uncertainty. These approaches show their inherent difficulties as pointed out by Molodtsov [6], because of intensive quantity and type of uncertainties. In Reference [6], Molodtsov defines the soft set which is a new logical instrument for dealing uncertainties.

Soft set theory attracts many authors because it has a vast range of applications in many areas like the smoothness of functions, decision making, probability theory, data analysis, measurement theory, forecasting and operations research [6-10]. Nowadays, many authors work to hybridize the different models with soft set and achieved results in many applicable theories. Maji defines the fuzzy soft set and intuitionistic fuzzy soft set [11,12]. Then the further extensions of soft sets like the generalized fuzzy soft set [13], the interval-valued fuzzy soft set [14], the soft rough set [15], the vague soft set [16], the trapezoidal fuzzy soft set [17], the neutrosophic soft set [18], the intuitionistic neutrosophic soft set [19], the multi-fuzzy soft set [20] and the hesitant fuzzy soft set [21] are introduced. Agarwal defines the generalized intuitionistic fuzzy soft set (GIFSS) [22] which has some problems pointed out by Feng [23] and redefined GIFSS.

In Reference [24], Coung defines the picture fuzzy set which is an extension of the fuzzy soft set and intuitionistic fuzzy set. In Reference [25], Sing defines the correlation coefficients of PFS and their applications in clustering analysis. In Reference [26], Son and Thong define time arrangement gauging and climate estimating with the help of novel fuzzy calculations based on the PFS domain. A novel fuzzy derivation framework on PFS to enhance the inference performance of the traditional fuzzy inference system is proposed by Son [27]. A novel picture fuzzy clustering technique is applied for complex data and particle swarm optimization by Thong [28]. Wei [29] defines the picture fuzzy aggregation operators method and uses them to multi attribute decision making (MADM) for ordering enterprise resource planning (ERP) structures. Wei [30], in the light of the picture fuzzy weighted cross-entropy a basic leadership technique is researched and used it to order the choices. In Reference [31], Garg defines aggregation operations on picture fuzzy soft set (PFSS) and used them to multi criteria decision-making (MCDM) problems. In Reference [32], Yang defined the picture fuzzy soft set and applied them to decision making problems. In Reference [33], Jabir khan defines the generalized picture fuzzy soft set and applied them to decision making problems. For more about decision making, we refer to References [34-38].

The soft matrix and its operations in a soft set are defined by Cagman [39]. The notion of soft discernibility matrix $(\mathcal{S D} \mathcal{M})$ is given by Feng and Zhou, which not only provide the best choice but also an order relation among all alternatives [40]. In [41], Feng defines an adjustable approach for the fuzzy soft set and an adjustable approach for the intuitionistic fuzzy soft set is defined by Jiang [42]. In Reference [32], an adjustable $\mathcal{S D M}$ is define dfor picture fuzzy soft sets.

The idea of GIFSS is very encouraging in decision-making since it considers how to capitalize an additional intuitionistic fuzzy input from the director to minimize any possible perversion in the data provided by evaluating specialists. First, Skowron and Rauszer [43] initiated the concept of discernibility matrix and extensively used in rough sets to solve attribute reduction, and its influence is significant and easy to understand. Also, in our daily life decision making problems, different attributes are not of equal importance. Some are more important than others, therefore, the decision maker assigns different values (weights) to different attributes and imposes different threshold functions when we need a restriction to a positive membership function and negative membership function. In this paper, we use an adjustable perspective to GIFSS and get level soft sets. Then each GIFSS can be seen as a level soft set and composed a crisp soft set, therefore, for solving decision making problems we apply $\mathcal{W} \mathcal{S} \mathcal{M}$. 
The purpose of this paper is to use weighted soft discernibility matrix $(\mathcal{W S D M})$ for GIFSS using an adjustable perspective to solve decision making problems. In the literature, the GIFSS set is defined and applied for decision making problems using intuitionistic fuzzy weighted averaging operators. This technique can not only give the best alternative but also an order relation of all alternatives easily by scanning the $\mathcal{W S D M}$ at most one time. In this paper, Sections 1 and 2 consists of an introduction and preliminaries which include the basic definition related to fuzzy sets, intuitionistic fuzzy set and $\mathcal{S D M}$. Section 3 is devoted to the threshold functions and their level soft sets. In Section 4, two algorithms are proposed basis on $\mathcal{W} \mathcal{S D M}$ to solve decision making problem using GIFSS. Section 5 consists of a case study of scholarship for a doctoral degree. Finally, comparison and conclusion are given in Sections 6 and 7.

\section{Preliminaries}

In this section, we present the basic definitions of fuzzy set, intuitionistic fuzzy set, soft set, generalized intuitionistic fuzzy soft set, soft discernibility matrix and weighted soft discernibility matrix.

Throughout this paper, finite set $\hat{\mathcal{Y}}=\left\{\ell_{1}, \ell_{2}, \ldots, \ell_{n}\right\}$ and $\hat{P}=\left\{\epsilon_{1}, \epsilon_{2}, \ldots, \epsilon_{m}\right\}$ represents the set of $n$ alternatives and $m$ attributes (parameters). The abbreviations IFS, IFSS, GIFSS, SDP, SDM and $\mathcal{W S D} \mathcal{M}$ represents the intuitionistic fuzzy set, intuitionistic fuzzy soft set, generalized intuitionistic fuzzy soft set, soft discernibility parameters, soft discernibility matrix and weighted soft discernibility matrix, respectively. Moreover, the abbreviation "w.r.t." is used for "with respect to."

A fuzzy set is defined by Zadeh [1], which handles uncertainty based on the view of gradualness effectively.

Definition 1 ([1]). A membership function $\xi_{\hat{\mathcal{A}}}: \hat{\mathcal{Y}} \rightarrow[0,1]$ defines the fuzzy set $\hat{\mathcal{A}}$ over the $\hat{\mathcal{Y}}$, where $\xi_{\hat{\mathcal{A}}}(\ell)$ particularized the membership of an element $\ell \in \hat{\mathcal{Y}}$ in fuzzy set $\hat{\mathcal{A}}$.

Like a membership degree on an element in a fuzzy set, human intuition suggest that there is a non-membership degree of an element in a set. In Reference [4], an IFS defined by Atanassov to sketch the imprecision of human beings when needing the judgments over the elements.

Definition 2 ([4]). An IFS $\hat{\mathcal{A}}$ over the universe $\hat{\mathcal{Y}}$ is defined as

$$
\hat{\mathcal{A}}=\left\{\left(\ell, \xi_{\hat{\mathcal{A}}}, \vartheta_{\hat{\mathcal{A}}}\right) \mid \ell \in \hat{\mathcal{Y}}\right\},
$$

where $\xi_{\hat{\mathcal{A}}}: \hat{\mathcal{Y}} \rightarrow[0,1]$ and $\vartheta_{\hat{\mathcal{A}}}: \hat{\mathcal{Y}} \rightarrow[0,1]$ are the degree of positive membership and degree of negative membership, respectively. Furthermore, it is required that $0 \leq \xi_{\hat{\mathcal{A}}}+\vartheta_{\hat{\mathcal{A}}} \leq 1$.

A soft set is defined by Molodtsov [6], which provides an effective framework to dealings with imprecision with the parametric point of view, that is, each element is judged by some criteria of attributes.

Definition 3 ([6]). Let $\hat{\mathcal{Y}}$ be a universal set, $\hat{P}$ a parameter space, $\hat{\mathcal{A}} \subseteq \hat{P}$ and $P(\hat{\mathcal{Y}})$ the power set of $\hat{\mathcal{Y}}$. A pair $(\hat{\mathcal{F}}, \hat{\mathcal{A}})$ is called a soft set over $\hat{\mathcal{Y}}$, where $\hat{\mathcal{F}}$ is a set valued mapping given by $\hat{\mathcal{F}}: \hat{\mathcal{A}} \rightarrow P(\hat{\mathcal{Y}})$.

In [12], Maji defines the IFSS as follows.

Definition 4 ([12]). Let $\hat{\mathcal{Y}}$ be a universal set, $\hat{P}$ a parameter space, $\hat{\mathcal{A}} \subset \hat{P}$ and $\operatorname{IF}(\hat{\mathcal{Y}})$ the set of all IFSs of $\hat{\mathcal{Y}}$. A pair $(\hat{\mathcal{F}}, \hat{\mathcal{A}})$ is called an IFSS over $\hat{\mathcal{Y}}$, where $\hat{\mathcal{F}}$ is a set valued mapping given by $\hat{\mathcal{F}}: \hat{\mathcal{A}} \rightarrow \operatorname{IF}(\hat{\mathcal{Y}})$. 
The idea of GIFSS is very encouraging in decision-making since it considers how to capitalize an additional intuitionistic fuzzy input from the director to minimize any possible perversion in the data provided by evaluating specialists. First Agarwal [22], defines the GIFSS, but it has some problems, therefore, in Reference [23], Feng redefines the GIFSS as follows.

Definition 5 ([23]). Let $\hat{\mathcal{Y}}$ be a universal set, $\hat{\mathcal{A}} \subset \hat{P}$ a parametric set. By a GIFSS we mean a triple $(\hat{\mathcal{F}}, \hat{\mathcal{A}}, \hat{\rho})$, where $(\hat{\mathcal{F}}, \hat{\mathcal{A}})$ is an IFSS over $\hat{\mathcal{Y}}$ and $\hat{\rho}: \hat{\mathcal{A}} \rightarrow \operatorname{IF}(\hat{\mathcal{A}})$ is an IFS in $\hat{\mathcal{A}}$.

where $(\hat{\mathcal{F}}, \hat{\mathcal{A}})$ is called basic intuitionistic fuzzy soft set (BIFSS) and $\hat{\rho}$ is called the parametric intuitionistic fuzzy set (PIFS).

In [40], Q. Feng defines the $\mathcal{S D} \mathcal{M}$ for soft sets which provide not only the best alternative but also an order relation among all the alternatives.

Definition 6 ([40]). Let $(\hat{\mathcal{F}}, \hat{\mathcal{A}})$ be a soft set over $\hat{\mathcal{Y}}$. $\hat{\mathcal{F}}$ determined the partition $U \mid \operatorname{IND}(\hat{\mathcal{F}}, \hat{\mathcal{A}})=\left\{N_{i}: i \leq|\hat{\mathcal{Y}}|\right\}$ of $\hat{\mathcal{Y}}$. The $\mathcal{S D} \mathcal{M}$ is defined as $\mathcal{M}=\left(M\left(N_{i}, N_{j}\right)\right)_{i, j \leq|\hat{\mathcal{Y}}|}$, where $M\left(N_{i}, N_{j}\right)$ is called the SDPs among $N_{i}$ and $N_{j}$ and defined as

$$
M\left(N_{i}, N_{j}\right)=\left\{\hat{E}^{i} \cup \hat{E}^{j}: i, j \leq|\hat{\mathcal{Y}}|\right\}
$$

In which

$$
\hat{E}^{i}=\left\{\epsilon_{p}^{i}: \hat{\mathcal{F}}\left(\ell_{i}, \epsilon_{p}\right)=1 \text { and } \hat{\mathcal{F}}\left(\ell_{j}, \epsilon_{p}\right)=0, \forall \ell_{i} \in N_{i}, \forall \ell_{j} \in N_{j}\right\}
$$

and

$$
\hat{E}^{j}=\left\{\epsilon_{p}^{j}: \hat{\mathcal{F}}\left(\ell_{j}, \epsilon_{p}\right)=1 \text { and } \hat{\mathcal{F}}\left(\ell_{i}, \epsilon_{p}\right)=0, \forall \ell_{j} \in N_{j}, \forall \ell_{i} \in N_{i}\right\}
$$

The symbol $\epsilon_{p}^{i}$ (or $\epsilon_{p}^{j}$ ) represents the elements in $N_{i}$ (or $N_{j}$ ) have the value 1 at the attribute $\epsilon_{p}$, that is, $\hat{\mathcal{F}}\left(\ell_{i}, \epsilon_{p}\right)=1, \ell_{i} \in N_{i}\left(\right.$ or $\left.\hat{\mathcal{F}}\left(\ell_{j}, \epsilon_{p}\right)=1, \ell_{j} \in N_{j}\right)$.

The $\mathcal{W S D} \mathcal{M}$ is defined as follows.

Definition 7 ([40]). Let $(\hat{\mathcal{F}}, \hat{\mathcal{A}})$ be a soft set over $\hat{\mathcal{Y}}$. $\hat{\mathcal{F}}$ determined the partition $U \mid \operatorname{IND}(\hat{\mathcal{F}}, \hat{\mathcal{A}})=\left\{N_{i}: i \leq|\hat{\mathcal{Y}}|\right\}$ of $\hat{\mathcal{Y}}$. The $\mathcal{W} \mathcal{S D} \mathcal{M}$ is defined as $\mathcal{M}=\left(M\left(N_{i}, N_{j}\right)\right)_{i, j \leq|\hat{\mathcal{Y}}|}$, where $M\left(N_{i}, N_{j}\right)$ is called the SDPs among $N_{i}$ and $N_{j}$ and defined as

$$
M\left(N_{i}, N_{j}\right)=\left\{\hat{E}^{i} \cup \hat{E}^{j}: i, j \leq|\hat{\mathcal{Y}}|\right\}
$$

In which

$$
\hat{E}^{i}=\left\{\epsilon_{p}^{i * \hat{\omega}_{i}}: \hat{\mathcal{F}}\left(\ell_{i}, \epsilon_{p}\right)=1 \text { and } \hat{\mathcal{F}}\left(\ell_{j}, \epsilon_{p}\right)=0, \forall \ell_{i} \in N_{i}, \forall \ell_{j} \in N_{j}\right\}
$$

and

$$
\hat{E}^{j}=\left\{\epsilon_{p}^{j * \hat{\omega}_{j}}: \hat{\mathcal{F}}\left(\ell_{j}, \epsilon_{p}\right)=1 \text { and } \hat{\mathcal{F}}\left(\ell_{i}, \epsilon_{p}\right)=0, \forall \ell_{j} \in N_{j}, \forall \ell_{i} \in N_{i}\right\}
$$

The symbol $\epsilon_{p}^{i * \hat{\omega}_{i}}$ (or $\epsilon_{p}^{j * \hat{\omega}_{j}}$ ) represents the elements in $N_{i}$ (or $\left.N_{j}\right)$ have the value 1 at the parameter $\epsilon_{p}$, that is, $\hat{\mathcal{F}}\left(\ell_{i}, \epsilon_{p}\right)=1, \ell_{i} \in N_{i}\left(\right.$ or $\left.\hat{\mathcal{F}}\left(\ell_{j}, \epsilon_{p}\right)=1, \ell_{j} \in N_{j}\right)$.

In [40], Q. Feng also give some properties of $\mathcal{S D M}$ and $\mathcal{W S D M}$. 
Proposition 1 ([40]). Let $(\hat{\mathcal{F}}, \hat{\mathcal{A}})$ be a soft set over $\hat{\mathcal{Y}}$, where $\hat{\mathcal{Y}}=\left\{\ell_{1}, \ell_{2}, \ldots, \ell_{n}\right\}$ and $\varphi\left(N_{i}, N_{j}\right)=\left|M\left(N_{i}, N_{j}\right)\right|$. Then the $\mathcal{S D M}$ has the following characteristics:

1: $\quad M\left(N_{i}, N_{i}\right)=\varnothing(\forall i \leq n)$;

2: $\quad M\left(N_{i}, N_{j}\right)=M\left(N_{j}, N_{i}\right)(\forall i, j \leq n)$;

3: $\varphi\left(N_{i}, N_{i}\right)=0(\forall i \leq n)$;

4: $\varphi\left(N_{i}, N_{j}\right)=\varphi\left(N_{j}, N_{i}\right)(\forall i, j \leq n)$;

5: $\quad \varphi\left(N_{i}, N_{j}\right)=\left|M\left(N_{i}, N_{j}\right)\right|=\left|\hat{E}^{i}\right|+\left|\hat{E}^{j}\right| ;$

6: If $\varphi\left(N_{i}, N_{j}\right)=2 m\left(m \in N^{+}\right)$and $\left|\hat{E}^{i}\right|=\left|\hat{E}^{j}\right|$ then the elements of $N_{i}$ and $N_{j}$ have same rank (order);

7: If $\varphi\left(N_{i}, N_{j}\right)=2 m+1\left(m \in N^{+}\right)$, then $\left|\hat{E}^{i}\right| \neq\left|\hat{E}^{j}\right|$, that is, $\left|\hat{E}^{i}\right|<\left|\hat{E}^{j}\right|$ or $\left|\hat{E}^{i}\right|>\left|\hat{E}^{j}\right|$ and there is an order relation between the elements of $N_{i}$ and $N_{j}$.

\section{Threshold Functions and Level Soft Sets}

Definition 8. Let $\hat{\Gamma}=(\hat{\mathcal{F}}, \hat{\mathcal{A}}, \hat{\rho})$ be a GIFSS over $\hat{\mathcal{Y}}$ and $\hat{\mathcal{A}} \subseteq \hat{P}$ be a set of attributes. For a triple $(k, l, \alpha)$, where $(k, l), \alpha \in[0,1]^{2}$, the $(k, l, \alpha)$-level soft set of $\hat{\Gamma}$ is a crisp soft $\operatorname{set} \mathcal{L}(\hat{\Gamma} ;(k, l, \alpha))=\left(\hat{\mathcal{F}}_{(k, l)}, \hat{\mathcal{A}}, \hat{\rho}_{\alpha}\right)$ defined by

1. $\mathcal{L}(\hat{\Gamma} ; k, l)=\left(\hat{\mathcal{F}}_{(k, l)}, \hat{\mathcal{A}}\right)$ is a $(k, l)$ level-soft set of an IFSS defined by

$$
\hat{\mathcal{F}}_{(k, l)}(\epsilon)=\mathcal{L}(\hat{\mathcal{F}}(\epsilon) ; k, l)=\left\{\ell \in \hat{\mathcal{Y}} \mid \xi_{\hat{\mathcal{F}}(\epsilon)}(\ell) \geq k \text { and } \vartheta_{\hat{\mathcal{F}}(\epsilon)}(\ell) \leq l\right\}, \text { for all } \epsilon \in \hat{\mathcal{A}} .
$$

2. $\hat{\rho}_{\alpha}$ is an $\alpha$-level soft set of an IFS defined by

$$
\hat{\rho}_{\alpha}=\mathcal{L}(\hat{\rho} ; \alpha)=\left\{\epsilon \in \hat{\mathcal{A}} \mid \xi_{\hat{\rho}(\epsilon)} \geq u \text { and } \vartheta_{\hat{\rho}(\epsilon)} \leq v\right\} \text {, where } \alpha=(u, v) \text {. }
$$

Example 1. Department appointment to higher positions in an institute and an applicant may be evaluated by criteria such as "creativity" and "managerial skills" and so forth.

Suppose that there are five candidates $\hat{\mathcal{Y}}=\left\{\ell_{1}, \ell_{2}, \ell_{3}, \ell_{4}, \ell_{5}\right\}$ for senior positions and $\hat{\mathcal{A}}=$ $\left\{\epsilon_{1}, \epsilon_{2}, \epsilon_{3}, \epsilon_{4}, \epsilon_{5}, \epsilon_{6}\right\}$ is a criteria for evaluating candidates, where each $\epsilon_{i}$ stands for "creativity", "managerial skills", "intuition", "research productivity", "ability to work under pressure" and "knowledge", respectively. Suppose that

$$
\begin{aligned}
& \hat{\mathcal{F}}\left(\epsilon_{1}\right)=\left\{(0.8,0.1) / \ell_{1},(0.6,0.2) / \ell_{2},(0.7,0.3) / \ell_{3},(0.8,0.1) / \ell_{4},(0.5,0.4) / \ell_{5}\right\}, \\
& \hat{\mathcal{F}}\left(\epsilon_{2}\right)=\left\{(0.6,0.2) / \ell_{1},(0.7,0.2) / \ell_{2},(0.8,0.1) / \ell_{3},(0.9,0.1) / \ell_{4},(0.6,0.3) / \ell_{5}\right\}, \\
& \hat{\mathcal{F}}\left(\epsilon_{3}\right)=\left\{(0.4,0.4) / \ell_{1},(0.4,0.5) / \ell_{2},(0.6,0.3) / \ell_{3},(0.6,0.2) / \ell_{4},(0.7,0.2) / \ell_{5}\right\}, \\
& \hat{\mathcal{F}}\left(\epsilon_{4}\right)=\left\{(0.7,0.2) / \ell_{1},(0.5,0.1) / \ell_{2},(0.7,0.1) / \ell_{3},(0.5,0.3) / \ell_{4},(0.2,0.6) / \ell_{5}\right\}, \\
& \hat{\mathcal{F}}\left(\epsilon_{5}\right)=\left\{(0.6,0.1) / \ell_{1},(0.6,0.2) / \ell_{2},(0.2,0.5) / \ell_{3},(0.7,0.2) / \ell_{4},(0.7,0.1) / \ell_{5}\right\}, \\
& \hat{\mathcal{F}}\left(\epsilon_{6}\right)=\left\{(0.7,0.3) / \ell_{1},(0.5,0.3) / \ell_{2},(0.7,0.1) / \ell_{3},(0.6,0.2) / \ell_{4},(0.3,0.5) / \ell_{5}\right\} .
\end{aligned}
$$

In addition, $\hat{\rho}$ is the PIFS which is given by

$$
\hat{\rho}=\left\{(0.4,0.2) / \epsilon_{1},(0.8,0.2) / \epsilon_{2},(0.5,0.3) / \epsilon_{3},(0.6,0.2) / \epsilon_{4},(0.7,0.1) / \epsilon_{5},(0.4,0.4) / \epsilon_{6}\right\} .
$$

The tabular representation of GIFSS $(\hat{\mathcal{F}}, \hat{\mathcal{A}}, \hat{\rho})$ is shown in Table 1. 
Table 1. The $\operatorname{GIFSS}(\hat{\mathcal{F}}, \hat{\mathcal{A}}, \hat{\rho})$.

\begin{tabular}{ccccccc}
\hline$\hat{\mathcal{Y}}$ & $\epsilon_{1}$ & $\epsilon_{2}$ & $\epsilon_{3}$ & $\epsilon_{4}$ & $\epsilon_{5}$ & $\epsilon_{\mathbf{6}}$ \\
\hline$\ell_{1}$ & $(0.8,0.1)$ & $(0.6,0.2)$ & $(0.4,0.4)$ & $(0.7,0.2)$ & $(0.6,0.1)$ & $(0.7,0.3)$ \\
$\ell_{2}$ & $(0.6,0.2)$ & $(0.7,0.2)$ & $(0.4,0.5)$ & $(0.5,0.1)$ & $(0.6,0.2)$ & $(0.5,0.3)$ \\
$\ell_{3}$ & $(0.7,0.3)$ & $(0.8,0.1)$ & $(0.6,0.3)$ & $(0.7,0.1)$ & $(0.2,0.5)$ & $(0.7,0.1)$ \\
$\ell_{4}$ & $(0.8,0.1)$ & $(0.9,0.1)$ & $(0.6,0.2)$ & $(0.5,0.3)$ & $(0.7,0.2)$ & $(0.6,0.2)$ \\
$\ell_{5}$ & $(0.5,0.4)$ & $(0.6,0.3)$ & $(0.7,0.2)$ & $(0.2,0.6)$ & $(0.7,0.1)$ & $(0.3,0.5)$ \\
\hline$\hat{\rho}$ & $(0.4,0.2)$ & $(0.8,0.2)$ & $(0.5,0.3)$ & $(0.6,0.2)$ & $(0.7,0.1)$ & $(0.4,0.4)$ \\
\hline
\end{tabular}

Now we take $k=0.6, l=0.3$ and $\alpha=(0.5,0.3)$, then we have the following:

$$
\begin{aligned}
& \mathcal{L}\left(\hat{\mathcal{F}}\left(\epsilon_{1}\right) ; 0.6,0.3\right)=\left\{\ell_{1}, \ell_{2}, \ell_{3}, \ell_{4}\right\}, \\
& \mathcal{L}\left(\hat{\mathcal{F}}\left(\epsilon_{2}\right) ; 0.6,0.3\right)=\left\{\ell_{1}, \ell_{2}, \ell_{3}, \ell_{4}, \ell_{5}\right\}, \\
& \mathcal{L}\left(\hat{\mathcal{F}}\left(\epsilon_{3}\right) ; 0.6,0.3\right)=\left\{\ell_{3}, \ell_{4}, \ell_{5}\right\}, \\
& \mathcal{L}\left(\hat{\mathcal{F}}\left(\epsilon_{4}\right) ; 0.6,0.3\right)=\left\{\ell_{1}, \ell_{3}\right\}, \\
& \mathcal{L}\left(\hat{\mathcal{F}}\left(\epsilon_{5}\right) ; 0.6,0.3\right)=\left\{\ell_{1}, \ell_{2}, \ell_{4}, \ell_{5}\right\}, \\
& \mathcal{L}\left(\hat{\mathcal{F}}\left(\epsilon_{6}\right) ; 0.6,0.3\right)=\left\{\ell_{1}, \ell_{3}, \ell_{4}\right\},
\end{aligned}
$$

and

$$
\mathcal{L}(\hat{\rho} ; 0.5,0.3)=\left\{\epsilon_{2}, \epsilon_{3}, \epsilon_{4}, \epsilon_{5}\right\}
$$

Hence the $(0.6,0.3,(0.5,0.3))$-level soft set of $\hat{\Gamma}=(\hat{\mathcal{F}}, \hat{\mathcal{A}}, \hat{\rho})$ is a soft set $L=(\hat{\Gamma} ; 0.6,0.3,(0.5,0.3))=$ $\left(\hat{\mathcal{F}}_{(0.6,0.3)}, \hat{\mathcal{A}}_{1} \hat{\rho}_{(0.5,0.3)}\right)$, where the set valued mappings $\hat{\mathcal{F}}_{(0.6,0.3)}: \hat{\mathcal{A}} \rightarrow P(\hat{\mathcal{Y}})$ and $\hat{\rho}_{(0.5,0.3)}: \hat{\mathcal{A}} \rightarrow P(\hat{\mathcal{A}})$ are defined as $\hat{\mathcal{F}}_{(0.6,0.3)}(\epsilon)=\mathcal{L}(\hat{\mathcal{F}} ; 0.6,0.3)$ for all $\epsilon \in \hat{\mathcal{A}}$ and $\hat{\rho}_{(0.5,0.3)}=\mathcal{L}(\hat{\rho} ;(0.5,0.3))$, where $P(\hat{\mathcal{Y}})$ and $P(\hat{\mathcal{A}})$ are power sets of $\hat{\mathcal{Y}}$ and $\hat{\mathcal{A}}$, respectively. The tabular representation of $(0.6,0.3,(0.5,0.3))$-level soft set is given in Table 2.

Table 2. $(0.6,0.3,(0.5,0.3))$-level soft set of $\hat{\Gamma}$.

\begin{tabular}{ccccccc}
\hline$\hat{\mathcal{Y}}$ & $\epsilon_{1}$ & $\epsilon_{2}$ & $\epsilon_{3}$ & $\epsilon_{4}$ & $\epsilon_{5}$ & $\epsilon_{\mathbf{6}}$ \\
\hline$\ell_{1}$ & 1 & 1 & 0 & 1 & 1 & 1 \\
$\ell_{2}$ & 1 & 1 & 0 & 0 & 1 & 0 \\
$\ell_{3}$ & 1 & 1 & 1 & 1 & 0 & 1 \\
$\ell_{4}$ & 1 & 1 & 1 & 0 & 1 & 1 \\
$\ell_{5}$ & 0 & 1 & 1 & 0 & 1 & 0 \\
\hline$\hat{\rho}$ & 0 & 1 & 1 & 1 & 1 & 0 \\
\hline
\end{tabular}

Now we show some properties of $(k, l, \alpha)$-level soft sets.

Theorem 1. Let $\hat{\Gamma}=(\hat{\mathcal{F}}, \hat{\mathcal{A}}, \hat{\rho})$ be a GIFSS over $\hat{\mathcal{Y}}$ and $\hat{\mathcal{A}} \subseteq \hat{P}$ a set of parameters. Let $\mathcal{L}\left(\hat{\Gamma} ; k_{1}, l_{1}, \alpha_{1}\right)$ and $\mathcal{L}\left(\hat{\Gamma} ; k_{2}, l_{2}, \alpha_{2}\right)$ are $\left(k_{1}, l_{1}, \alpha_{1}\right)$ and $\left(k_{2}, l_{2}, \alpha_{2}\right)$-level soft sets of $\hat{\Gamma}$, respectively, where $k_{1}, l_{1}, k_{2}, l_{2} \in[0,1]$ and $\alpha_{1}, \alpha_{2} \in[0,1]^{2}$. If $k_{1} \geq k_{2}, l_{2} \geq l_{1}$ and $\alpha_{2} \subseteq \alpha_{1}$, then we have

$$
\mathcal{L}\left(\hat{\Gamma} ; k_{1}, l_{1}, \alpha_{1}\right) \subseteq \mathcal{L}\left(\hat{\Gamma} ; k_{2}, l_{2}, \alpha_{2}\right) .
$$

Proof. To complete the proof, we need to show

1. $\mathcal{L}\left(\hat{\Gamma} ; k_{1}, l_{1}\right) \subseteq \mathcal{L}\left(\hat{\Gamma} ; k_{2}, l_{2}\right)$ ， 
2. $\mathcal{L}\left(\hat{\rho} ; \alpha_{1}\right) \subseteq \mathcal{L}\left(\hat{\rho} ; \alpha_{2}\right)$.

Let $\mathcal{L}\left(\hat{\Gamma} ; k_{1}, l_{1}\right)=\left(\hat{\mathcal{F}}_{\left(k_{1}, l_{1}\right)}, \hat{\mathcal{A}}\right)$, where $\hat{\mathcal{F}}_{\left(k_{1}, l_{1}\right)}(\epsilon)=\left\{\ell \in \hat{\mathcal{Y}} \mid \xi_{\hat{\mathcal{F}}(\epsilon)}(\ell) \geq k_{1}\right.$ and $\left.\vartheta_{\hat{\mathcal{F}}(\epsilon)}(\ell) \leq l_{1}\right\}$ and $\mathcal{L}\left(\hat{\Gamma} ; k_{2}, l_{2}\right)=\left(\hat{\mathcal{F}}_{\left(k_{2}, l_{2}\right)}, \hat{\mathcal{A}}\right)$, where $\hat{\mathcal{F}}_{\left(k_{2}, l_{2}\right)}(\epsilon)=\left\{\ell \in \hat{\mathcal{Y}} \mid \xi_{\hat{\mathcal{F}}(\epsilon)}(\ell) \geq k_{2}\right.$ and $\left.\vartheta_{\hat{\mathcal{F}}(\epsilon)}(\ell) \leq l_{2}\right\}$ for all $\epsilon \in \hat{\mathcal{A}}$ are the $\left(k_{1}, l_{1}\right)$ and $\left(k_{2}, l_{2}\right)$-level soft sets of an IFSS.

Since $k_{1} \geq k_{2}$ and $l_{2} \geq l_{1}$, then for all $\epsilon \in \hat{\mathcal{A}}$ we have the following

$$
\begin{aligned}
\left\{\ell \in \hat{\mathcal{Y}} \mid \xi_{\hat{\mathcal{F}}(\epsilon)}(\ell) \geq k_{1} \text { and } \vartheta_{\hat{\mathcal{F}}(\epsilon)}(\ell) \leq l_{1}\right\} & \subseteq\left\{\ell \in \hat{\mathcal{Y}} \mid \xi_{\hat{\mathcal{F}}(\epsilon)}(\ell) \geq k_{2} \text { and } \vartheta_{\hat{\mathcal{F}}(\epsilon)}(\ell) \leq l_{2}\right\} \\
\hat{\mathcal{F}}_{\left(k_{1}, l_{1}\right)}(\epsilon) & \subseteq \hat{\mathcal{F}}_{\left(k_{2}, l_{2}\right)}(\epsilon) \\
\mathcal{L}\left(\hat{\Gamma}_{\boldsymbol{\Gamma}_{1}}, l_{1}\right) & \subseteq \mathcal{L}\left(\hat{\Gamma} ; k_{2}, l_{2}\right) .
\end{aligned}
$$

Let $\alpha_{1}=\left(u_{1}, v_{1}\right)$ and $\alpha_{2}=\left(u_{2}, v_{2}\right)$. Since $\alpha_{2} \subseteq \alpha_{1}$, therefore, $u_{2} \leq u_{1}$ and $v_{2} \geq v_{1}$. We have

$$
\begin{aligned}
\left\{\epsilon \in \hat{\mathcal{A}} \mid \xi_{\hat{\rho}(\epsilon)} \geq u_{1} \text { and } \vartheta_{\hat{\rho}(\epsilon)} \leq v_{1}\right\} & \subseteq\left\{\epsilon \in \hat{\mathcal{A}} \mid \xi_{\hat{\rho}(\epsilon)} \geq u_{2} \text { and } \vartheta_{\hat{\rho}(\epsilon)} \leq v_{2}\right\} \\
\mathcal{L}\left(\hat{\rho} ; \alpha_{1}\right) & \subseteq \mathcal{L}\left(\hat{\rho} ; \alpha_{2}\right) .
\end{aligned}
$$

Hence we have $\mathcal{L}\left(\hat{\Gamma} ; k_{1}, l_{1}, \alpha_{1}\right) \subseteq \mathcal{L}\left(\hat{\Gamma} ; k_{2}, l_{2}, \alpha_{2}\right)$.

Theorem 2. Let $\hat{\Gamma}_{1}=(\hat{\mathcal{F}}, \hat{\mathcal{A}}, \hat{\rho})$ and $\hat{\Gamma}_{2}=(\hat{\mathcal{G}}, \hat{\mathcal{A}}, \hat{\sigma})$ be GIFSSs over $\hat{\mathcal{Y}}$ and $\hat{\mathcal{A}} \subseteq \hat{P}$ a set of parameters. Let $\mathcal{L}\left(\hat{\Gamma}_{1} ; k, l, \alpha\right)$ and $\mathcal{L}\left(\hat{\Gamma}_{2} ; k, l, \alpha\right)$ are $(k, l, \alpha)$-level soft sets of $\hat{\Gamma}_{1}$ and $\hat{\Gamma}_{2}$, respectively, where $(k, l), \alpha \in[0,1]^{2}$. If $\hat{\Gamma}_{1} \subseteq \hat{\Gamma}_{2}$, then we have $\mathcal{L}\left(\hat{\Gamma}_{1} ; k, l, \alpha\right) \subseteq \mathcal{L}\left(\hat{\Gamma}_{2} ; k, l, \alpha\right)$.

Proof. Let $\mathcal{L}\left(\hat{\Gamma}_{1} ; k, l, \alpha\right)=\left(\hat{\mathcal{F}}_{(k, l)}, \hat{\mathcal{A}}, \hat{\rho}_{\alpha}\right)$ and $\mathcal{L}\left(\hat{\Gamma}_{2} ; k, l, \alpha\right)=\left(\hat{\mathcal{G}}_{(k, l)}, \hat{\mathcal{A}}, \hat{\sigma}_{\alpha}\right)$. Obviously, $\hat{\mathcal{A}} \subseteq \hat{\mathcal{A}}$. In the following we will prove that

1. $\mathcal{L}\left(\hat{\Gamma}_{1} ; k, l\right) \subseteq \mathcal{L}\left(\hat{\Gamma}_{2} ; k, l\right)$,

2. $\mathcal{L}(\hat{\rho} ; \alpha) \subseteq \mathcal{L}(\hat{\sigma} ; \alpha)$.

Let $\mathcal{L}\left(\hat{\Gamma}_{1} ; k, l\right)=\left(\hat{\mathcal{F}}_{(k, l)}, \hat{\mathcal{A}}\right)$, where $\hat{\mathcal{F}}_{(k, l)}(\epsilon)=\left\{\ell \in \hat{\mathcal{Y}} \mid \xi_{\hat{\mathcal{F}}_{(\epsilon)}}(\ell) \geq k\right.$ and $\left.\vartheta_{\hat{\mathcal{F}}_{(\epsilon)}}(\ell) \leq l\right\}$ and $\mathcal{L}\left(\hat{\Gamma}_{2} ; k, l\right)=\left(\hat{\mathcal{G}}_{(k, l)}, \hat{\mathcal{A}}\right)$, where $\hat{\mathcal{G}}_{(k, l)}(\epsilon)=\left\{\ell \in \hat{\mathcal{Y}} \mid \xi_{\hat{\mathcal{G}}(\epsilon)}(\ell) \geq k\right.$ and $\left.\vartheta_{\hat{\mathcal{G}}(\epsilon)}(\ell) \leq l\right\}$ for all $\epsilon \in \hat{\mathcal{A}}$ are the $(k, l)$-level soft sets of an IFSS. Assume that $\ell \in \hat{\mathcal{F}}_{(k, l)}(\epsilon)$. Since $\hat{\mathcal{F}}_{(k, l)}(\epsilon)=\left\{\ell \in \hat{\mathcal{Y}} \mid \xi_{\hat{\mathcal{F}}_{(\epsilon)}}(\ell) \geq\right.$ $k$ and $\left.\vartheta_{\hat{\mathcal{F}}(\epsilon)}(\ell) \leq l\right\}$, then we have $\xi_{\hat{\mathcal{F}}(\epsilon)}(\ell) \geq k$ and $\vartheta_{\hat{\mathcal{F}}(\epsilon)}(\ell) \leq l$. Since $\hat{\Gamma}_{1} \subseteq \hat{\Gamma}_{2}$, we have

$$
\xi_{\hat{\mathcal{G}}(\epsilon)}(\ell) \geq \xi_{\hat{\mathcal{F}}(\epsilon)}(\ell) \text { and } \vartheta_{\hat{\mathcal{G}}(\epsilon)}(\ell) \leq \vartheta_{\hat{\mathcal{F}}(\epsilon)}(\ell)
$$

Hence $\ell \in \hat{\mathcal{G}}_{(k, l)}(\epsilon)$, where $\hat{\mathcal{G}}_{(k, l)}(\epsilon)=\left\{\ell \in \hat{\mathcal{Y}} \mid \xi_{\hat{\mathcal{G}}(\epsilon)}(\ell) \geq k\right.$ and $\left.\vartheta_{\hat{\mathcal{G}}(\epsilon)}(\ell) \leq l\right\}$, because $\xi_{\hat{\mathcal{G}}_{(\epsilon)}}(\ell) \geq$ $\xi_{\hat{\mathcal{F}}(\epsilon)}(\ell) \geq k$ and $\vartheta_{\hat{\mathcal{G}}(\epsilon)}(\ell) \leq \vartheta_{\hat{\mathcal{F}}(\epsilon)}(\ell) \leq l$. This completes the first part, that is,

$$
\mathcal{L}\left(\hat{\Gamma}_{1} ; k, l\right) \subseteq \mathcal{L}\left(\hat{\Gamma}_{2} ; k, l\right) .
$$

Now, since $\hat{\rho} \subseteq \hat{\sigma}$, then for all $\epsilon \in \hat{\mathcal{A}}$ we have the following

$$
\xi_{\hat{\rho}(\epsilon)} \leq \xi_{\hat{\sigma}(\epsilon)} \text { and } \vartheta_{\hat{\rho}(\epsilon)} \geq \vartheta_{\hat{\sigma}(\epsilon)}
$$

Let $\alpha=(u, v)$ and

$$
\mathcal{L}(\hat{\rho} ; \alpha)=\left\{\epsilon \in \hat{\mathcal{A}} \mid \xi_{\hat{\rho}(\epsilon)} \geq u \text { and } \vartheta_{\hat{\rho}(\epsilon)} \leq v\right\},
$$

and

$$
\mathcal{L}(\hat{\sigma} ; \alpha)=\left\{\epsilon \in \hat{\mathcal{A}} \mid \xi_{\hat{\sigma}(\epsilon)} \geq u \text { and } \vartheta_{\hat{\sigma}(\epsilon)} \leq v\right\}
$$


Assume that $\epsilon \in \mathcal{L}(\hat{\rho} ; \alpha)$, then we have $\xi_{\hat{\rho}(\epsilon)} \geq u$ and $\vartheta_{\hat{\rho}(\epsilon)} \leq v$. Hence $\epsilon \in \mathcal{L}(\hat{\sigma} ; \alpha)$, because $\xi_{\hat{\sigma}(\epsilon)} \geq$ $\xi_{\hat{\rho}(\epsilon)} \geq u$ and $\vartheta_{\hat{\sigma}(\epsilon)} \leq \vartheta_{\hat{\rho}(\epsilon)} \leq v$, and we have

$$
\mathcal{L}(\hat{\rho} ; \alpha) \subseteq \mathcal{L}(\hat{\sigma} ; \alpha)
$$

Consequently, $\mathcal{L}\left(\hat{\Gamma}_{1} ; k, l, \alpha\right) \subseteq \mathcal{L}\left(\hat{\Gamma}_{2} ; k, l, \alpha\right)$.

Definition 9. Let $\hat{\Gamma}=(\hat{\mathcal{F}}, \hat{\mathcal{A}}, \hat{\rho})$ be a GIFSS over $\hat{\mathcal{Y}}$ and $\hat{\mathcal{A}} \subseteq \hat{P}$ be a set of attributes. Then the threshold function $\psi: \hat{\mathcal{A}} \rightarrow[0,1]^{2}$, i.e., $\psi(\epsilon)=(k(\epsilon), l(\epsilon)), \psi_{\hat{\rho}}=\left(u_{\psi}, v_{\psi}\right)$ and $k(\epsilon), l(\epsilon), u_{\psi}, v_{\psi} \in[0,1]$ for all $\epsilon \in \hat{\mathcal{A}}$. The level soft set of $\hat{\Gamma}$ w.r.t. $\psi$ is a crisp soft set $\mathcal{L}(\hat{\Gamma} ; \psi)=\left(\hat{\mathcal{F}}_{\psi}, \hat{\mathcal{A}}, \hat{\rho}_{\psi}\right)$ defined by $\mathcal{L}(\hat{\Gamma} ; \psi(\epsilon))=\left(\hat{\mathcal{F}}_{\psi(\epsilon)}, \hat{\mathcal{A}}\right)$, where for all $\epsilon \in \hat{\mathcal{A}}$

$$
\hat{\mathcal{F}}_{\psi}(\epsilon)=\mathcal{L}(\hat{\mathcal{F}}(\epsilon) ; \psi(\epsilon))=\left\{\ell \in \hat{\mathcal{Y}} \mid \xi_{\hat{\mathcal{F}}(\epsilon)}(\ell) \geq k(\epsilon) \text { and } \vartheta_{\hat{\mathcal{F}}(\epsilon)}(\ell) \leq l(\epsilon)\right\}
$$

and

$$
\hat{\rho}_{\psi}=\mathcal{L}(\hat{\rho} ; \psi)=\left\{\epsilon \in \hat{\mathcal{A}} \mid \xi_{\hat{\rho}(\epsilon)} \geq u_{\psi} \text { and } \vartheta_{\hat{\rho}(\epsilon)} \leq v_{\psi}\right\} .
$$

Definition 10. Let $\hat{\Gamma}=(\hat{\mathcal{F}}, \hat{\mathcal{A}}, \hat{\rho})$ be a GIFSS over $\hat{\mathcal{Y}}$ and $\hat{\mathcal{A}} \subseteq \hat{P}$ be a set of attributes. Then mid-level threshold function $\operatorname{mid}_{\hat{\Gamma}}: \hat{\mathcal{A}} \rightarrow[0,1]^{2}$, i.e., $\operatorname{mid}_{\hat{\Gamma}}(\epsilon)=\left(k_{\text {mid }_{\hat{\Gamma}}}(\epsilon), l_{\text {mid }_{\hat{\Gamma}}}(\epsilon)\right)$ and $\operatorname{mid}_{\hat{\Gamma}}(\hat{\rho})=\left(u_{\text {midd }_{\hat{\Gamma}}}, v_{\text {mid }_{\hat{\Gamma}}}\right)$ for all $\epsilon \in \hat{\mathcal{A}}$, where

$$
\begin{gathered}
k_{\text {mid }_{\hat{\Gamma}}}(\epsilon)=\frac{1}{|\hat{\mathcal{Y}}|} \sum_{\ell \in \hat{\mathcal{Y}}} \xi_{\hat{\mathcal{F}}(\epsilon)}(\ell) \text { and } l_{\text {mid }_{\hat{\Gamma}}}(\epsilon)=\frac{1}{|\hat{\mathcal{Y}}|} \sum_{\ell \in \hat{\mathcal{Y}}} \vartheta_{\hat{\mathcal{F}}(\epsilon)}(\ell), \\
u_{\operatorname{mid}_{\hat{\Gamma}}}=\frac{1}{|\hat{\mathcal{A}}|} \sum_{\epsilon \in \hat{\mathcal{A}}} \xi_{\hat{\rho}}(\epsilon) \text { and } v_{\operatorname{mid}_{\hat{\Gamma}}}=\frac{1}{|\hat{\mathcal{A}}|} \sum_{\epsilon \in \hat{\mathcal{A}}} \vartheta_{\hat{\rho}}(\epsilon) .
\end{gathered}
$$

$\mathcal{L}(\hat{\Gamma} ;$ mid $)$ is a notation used to represents the corresponding level soft set of $\hat{\Gamma}$ and is called the mid-level soft set of $\hat{\Gamma}$.

Definition 11. Let $\hat{\Gamma}=(\hat{\mathcal{F}}, \hat{\mathcal{A}}, \hat{\rho})$ be a GIFSS over $\hat{\mathcal{Y}}$ and $\hat{\mathcal{A}} \subseteq \hat{P}$ be a set of attributes. Then top-bottom-level threshold function $t b_{\hat{\Gamma}}: \hat{\mathcal{A}} \rightarrow[0,1]^{2}$, i.e., $t b_{\hat{\Gamma}}(\epsilon)=\left(k_{t b_{\hat{\Gamma}}}(\epsilon), l_{t b_{\hat{\Gamma}}}(\epsilon)\right)$ and $t b_{\hat{\Gamma}}(\hat{\rho})=\left(u_{t b_{\hat{\Gamma}}}, v_{t b_{\hat{\Gamma}}}\right)$ for all $\epsilon \in \hat{\mathcal{A}}$, where

$$
\begin{gathered}
k_{t b_{\hat{\Gamma}}}(\epsilon)=\max _{\ell \in \hat{\mathcal{Y}}} \xi_{\hat{\mathcal{F}}(\epsilon)}(\ell) \text { and } l_{t b_{\hat{\Gamma}}}(\epsilon)=\min _{\ell \in \hat{\mathcal{Y}}} \vartheta_{\hat{\mathcal{F}}(\epsilon)}(\ell), \\
u_{t b_{\hat{\Gamma}}}=\max _{\epsilon \in \hat{\mathcal{A}}} \xi_{\hat{\rho}}(\epsilon) \text { and } v_{t b_{\hat{\Gamma}}}=\min _{\epsilon \in \hat{\mathcal{A}}} \hat{\vartheta}_{\hat{\rho}}(\epsilon) .
\end{gathered}
$$

$\mathcal{L}(\hat{\Gamma} ; t b)$ is a notation used to represents the corresponding level soft set of $\hat{\Gamma}$ and called the top-bottom-level soft set of $\hat{\Gamma}$.

Definition 12. Let $\hat{\Gamma}=(\hat{\mathcal{F}}, \hat{\mathcal{A}}, \hat{\rho})$ be a GIFSS over $\hat{\mathcal{Y}}$ and $\hat{\mathcal{A}} \subseteq \hat{P}$ be a set of attributes. Then top-top-level threshold function $t t_{\hat{\Gamma}}: \hat{\mathcal{A}} \rightarrow[0,1]^{2}$, i.e., $t t_{\hat{\Gamma}}(\epsilon)=\left(k_{t t_{\hat{\Gamma}}}(\epsilon), l_{t t_{\hat{\Gamma}}}(\epsilon)\right)$ and $t t_{\hat{\Gamma}}(\hat{\rho})=\left(u_{t t_{\hat{\Gamma}}}, v_{t t_{\hat{\Gamma}}}\right)$ for all $\epsilon \in \hat{\mathcal{A}}$, where

$$
\begin{gathered}
k_{t t_{\hat{\Gamma}}}(\epsilon)=\max _{\ell \in \hat{\mathcal{Y}}} \xi_{\hat{\mathcal{F}}(\epsilon)}(\ell) \text { and } l_{t t_{\hat{\Gamma}}}(\epsilon)=\max _{\ell \in \hat{\mathcal{Y}}} \vartheta_{\hat{\mathcal{F}}(\epsilon)}(\ell), \\
u_{t t_{\hat{\Gamma}}}=\max _{\epsilon \in \hat{\mathcal{A}}} \xi_{\hat{\rho}}(\epsilon) \text { and } v_{t t_{\hat{\Gamma}}}=\max _{\epsilon \in \hat{\mathcal{A}}} \vartheta_{\hat{\rho}}(\epsilon) .
\end{gathered}
$$

$\mathcal{L}(\hat{\Gamma} ; t t)$ is a notation used to represents the corresponding level soft set of $\hat{\Gamma}$ and called the top-top-level soft set of $\hat{\Gamma}$. 
Definition 13. Let $\hat{\Gamma}=(\hat{\mathcal{F}}, \hat{\mathcal{A}}, \hat{\rho})$ be a GIFSS over $\hat{\mathcal{Y}}$ and $\hat{\mathcal{A}} \subseteq \hat{P}$ be a set of attributes. Then bottom-bottom-level threshold function $b b_{\hat{\Gamma}}: \hat{\mathcal{A}} \rightarrow[0,1]^{2}$, i.e., $b b_{\hat{\Gamma}}(\epsilon)=\left(k_{b b_{\hat{\Gamma}}}(\epsilon), l_{b b_{\hat{\Gamma}}}(\epsilon)\right)$ and $b b_{\hat{\Gamma}}(\hat{\rho})=\left(u_{b b_{\hat{\Gamma}}}, v_{b b_{\hat{\Gamma}}}\right)$ for all $\epsilon \in \hat{\mathcal{A}}$, where

$$
\begin{gathered}
k_{b b_{\hat{\Gamma}}}(\epsilon)=\min _{\ell \in \hat{\mathcal{Y}}} \xi_{\hat{\mathcal{F}}(\epsilon)}(\ell) \text { and } l_{b b_{\hat{\Gamma}}}(\epsilon)=\min _{\ell \in \hat{\mathcal{Y}}} \vartheta_{\hat{\mathcal{F}}(\epsilon)}(\ell), \\
u_{b b_{\hat{\Gamma}}}=\min _{\epsilon \in \hat{\mathcal{A}}} \xi_{\hat{\rho}}(\epsilon) \text { and } v_{b b_{\hat{\Gamma}}}=\min _{\epsilon \in \hat{\mathcal{A}}} \vartheta_{\hat{\rho}}(\epsilon) .
\end{gathered}
$$

$\mathcal{L}(\hat{\Gamma} ; b b)$ is a notation used to represents the corresponding level soft set of $\hat{\Gamma}$ and is called the bottom-bottom-level soft set of $\hat{\Gamma}$.

Definition 14. Let $\hat{\Gamma}=(\hat{\mathcal{F}}, \hat{\mathcal{A}}, \hat{\rho})$ be a GIFSS over $\hat{\mathcal{Y}}$ and $\hat{\mathcal{A}} \subseteq \hat{P}$ be a set of attributes. Then med-level threshold function $\operatorname{med}_{\hat{\Gamma}}: \hat{\mathcal{A}} \rightarrow[0,1]^{2}$, that is, $\operatorname{med}_{\hat{\Gamma}}(\epsilon)=\left(k_{\text {med }_{\hat{\Gamma}}}(\epsilon), l_{\text {med }_{\hat{\Gamma}}}(\epsilon)\right)$ and $\operatorname{med}_{\hat{\Gamma}}(\hat{\rho})=\left(u_{\text {med }_{\hat{\Gamma}}}, v_{\text {med }}\right)$ for all $\epsilon \in \hat{\mathcal{A}}$, where for all $\epsilon \in \hat{\mathcal{A}}$, if we align ascendingly (or descendingly) the value of membership function of all elements of BIFSS, $k_{\text {med }_{\hat{\Gamma}}}(\epsilon)$ represents the median, namely

$$
k_{\text {med }_{\hat{\Gamma}}}(\epsilon)= \begin{cases}\xi_{\hat{\mathcal{F}}(\epsilon)}\left(\ell_{\left(\frac{\mid \hat{Y}}{2} \mid+1\right.}\right), & \text { if }|\hat{\mathcal{Y}}| \text { is odd }, \\ \left(\xi_{\hat{\mathcal{F}}(\epsilon)}\left(\ell_{\left(\frac{\mid \hat{\mathcal{Y}}}{2}\right)}\right)+\right. & \\ \left.\xi_{\hat{\mathcal{F}}(\epsilon)}\left(\ell_{\left(\frac{\mid \hat{Y}}{2}+1\right)}\right)\right) / 2, & \text { if }|\hat{\mathcal{Y}}| \text { is even, }\end{cases}
$$

if we align ascendingly (or descendingly) the value of non-membership function of all elements of BIFSS, $l_{\text {med }}(\epsilon)$ represents the median, namely

$$
l_{\text {med }_{\hat{\Gamma}}}(\epsilon)= \begin{cases}\vartheta_{\hat{\mathcal{F}}(\epsilon)}\left(\ell_{\left(\frac{|\hat{\mathcal{Y}}|+1}{2}\right)}\right), & \text { if }|\hat{\mathcal{Y}}| \text { is odd, } \\ \left(\vartheta_{\hat{\mathcal{F}}(\epsilon)}\left(\ell_{\left(\frac{\mid \hat{\mathcal{Y}}}{2}\right)}\right)+\right. & \\ \left.\vartheta_{\hat{\mathcal{F}}(\epsilon)}\left(\ell_{\left(\frac{|\hat{\mathcal{Y}}|}{2}+1\right)}\right)\right) / 2, & \text { if }|\hat{\mathcal{Y}}| \text { is even, }\end{cases}
$$

if we align ascendingly (or descendingly) the value of membership function of all elements of PIFS, $u_{\text {med }}$ represents $_{\Gamma}$ the median, namely

$$
u_{\text {med }_{\hat{\Gamma}}}(\epsilon)= \begin{cases}\xi_{\hat{\rho}(\epsilon)}\left(\epsilon_{\left(\frac{|\hat{\mathcal{A}}|+1}{2}\right)}\right), & \text { if }|\hat{\mathcal{A}}| \text { is odd, } \\ \left(\xi_{\hat{\rho}(\epsilon)}\left(\epsilon_{\left(\frac{|\hat{\mathcal{A}}|}{2}\right)}\right)+\right. & \\ \left.\xi_{\hat{\rho}(\epsilon)}\left(\epsilon_{\left(\frac{|\mathcal{A}|}{2}+1\right)}\right)\right) / 2, & \text { if }|\hat{\mathcal{A}}| \text { is even, }\end{cases}
$$

and if we align ascendingly (or descendingly) the value of non-membership function of all elements of PIFS, $v_{\text {med }_{\hat{\Gamma}}}$ represents the median, namely

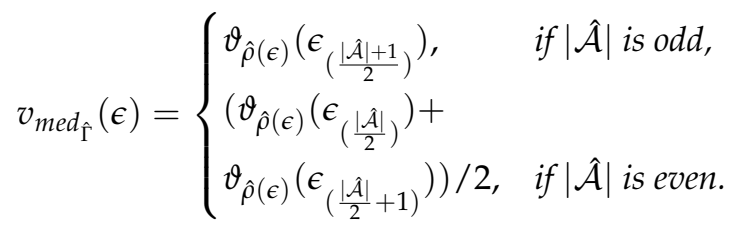

$\mathcal{L}(\hat{\Gamma} ;$ med $)$ is a notation used to represents the corresponding level soft set of $\hat{\Gamma}$ and called the med-level soft set of $\hat{\Gamma}$.

Example 2. Let us consider the GIFSS $\hat{\Gamma}=(\hat{\mathcal{F}}, \hat{\mathcal{A}}, \hat{\rho})$ in Example 1. The threshold functions mentioned above and their level soft sets with tabular representation (Tables 3-7) are given as follows. 
i. $\operatorname{mid}_{\hat{\Gamma}}=\left\{(0.68,0.22) / \epsilon_{1},(0.72,0.18) / \epsilon_{2},(0.54,0.32) / \epsilon_{3},(0.52,0.26) / \epsilon_{4},(0.56,0.22) / \epsilon_{5},(0.56,0.28) / \epsilon_{6}\right\}$, $\operatorname{mid}_{\hat{\Gamma}}(\hat{\rho})=(0.57,0.23)$.

The mid-level soft set is given as

$$
\begin{aligned}
\mathcal{L}(\hat{\mathcal{F}} ; \text { mid }) & =\left\{\left\{\ell_{1}, \ell_{4}\right\} / \epsilon_{1},\left\{\ell_{3}, \ell_{4}\right\} / \epsilon_{2},\left\{\ell_{3}, \ell_{4}, \ell_{5}\right\} / \epsilon_{3},\left\{\ell_{1}, \ell_{3}\right\} / \epsilon_{4},\left\{\ell_{1}, \ell_{2}, \ell_{4}, \ell_{5}\right\} / \epsilon_{5},\left\{\ell_{3}, \ell_{4}\right\} / \epsilon_{6},\right\}, \\
\mathcal{L}(\hat{\rho}, \text { mid }) & =\left\{\epsilon_{2}, \epsilon_{4}, \epsilon_{5}\right\} .
\end{aligned}
$$

ii. $t b_{\hat{\Gamma}}=\left\{(0.8,0.1) / \epsilon_{1},(0.9,0.1) / \epsilon_{2},(0.7,0.2) / \epsilon_{3},(0.7,0.1) / \epsilon_{4},(0.7,0.1) / \epsilon_{5},(0.7,0.1) / \epsilon_{6}\right\}$, $t b_{\hat{\Gamma}}(\hat{\rho})=(0.8,0.1)$.

The tb-level soft set is given as

$$
\begin{aligned}
\mathcal{L}(\hat{\mathcal{F}} ; t b) & =\left\{\left\{\ell_{1}, \ell_{4}\right\} / \epsilon_{1},\left\{\ell_{4}\right\} / \epsilon_{2},\left\{\ell_{5}\right\} / \epsilon_{3},\left\{\ell_{3}\right\} / \epsilon_{4},\left\{\ell_{5}\right\} / \epsilon_{5},\left\{\ell_{3}\right\} / \epsilon_{6}\right\} \\
\mathcal{L}(\hat{\rho}, t b) & =\{\} .
\end{aligned}
$$

iii. $t t_{\hat{\Gamma}}=\left\{(0.8,0.4) / \epsilon_{1},(0.9,0.3) / \epsilon_{2},(0.7,0.5) / \epsilon_{3},(0.7,0.6) / \epsilon_{4},(0.7,0.5) / \epsilon_{5},(0.7,0.5) / \epsilon_{6}\right\}$, $t t_{\hat{\Gamma}}(\hat{\rho})=(0.8,0.4)$.

The tt-level soft set is given as

$$
\begin{aligned}
\mathcal{L}(\hat{\mathcal{F}} ; t t) & =\left\{\left\{\ell_{1}, \ell_{4}\right\} / \epsilon_{1},\left\{\ell_{4}\right\} / \epsilon_{2},\left\{\ell_{5}\right\} / \epsilon_{3},\left\{\ell_{1}, \ell_{3}\right\} / \epsilon_{4},\left\{\ell_{4}, \ell_{5}\right\} / \epsilon_{5},\left\{\ell_{1}, \ell_{3}\right\} / \epsilon_{6}\right\}, \\
\mathcal{L}(\hat{\rho}, t t) & =\left\{\epsilon_{2}\right\} .
\end{aligned}
$$

iv. $b b_{\hat{\Gamma}}=\left\{(0.5,0.1) / \epsilon_{1},(0.6,0.1) / \epsilon_{2},(0.4,0.2) / \epsilon_{3},(0.2,0.1) / \epsilon_{4},(0.2,0.1) / \epsilon_{5},(0.3,0.1) / \epsilon_{6}\right\}$,

$b b_{\hat{\Gamma}}(\hat{\rho})=(0.4,0.1)$.

The bb-level soft set is given as

$$
\begin{aligned}
\mathcal{L}(\hat{\mathcal{F}} ; b b) & =\left\{\left\{\ell_{1}, \ell_{4}\right\} / \epsilon_{1},\left\{\ell_{3}, \ell_{4}\right\} / \epsilon_{2},\left\{\ell_{4}, \ell_{5}\right\} / \epsilon_{3},\left\{\ell_{2}, \ell_{3}\right\} / \epsilon_{4},\left\{\ell_{1}, \ell_{5}\right\} / \epsilon_{5},\left\{\ell_{3}\right\} / \epsilon_{6}\right\}, \\
\mathcal{L}(\hat{\rho}, b b) & =\left\{\epsilon_{5}\right\} .
\end{aligned}
$$

v. $\operatorname{med}_{\hat{\Gamma}}=\left\{(0.7,0.2) / \epsilon_{1},(0.7,0.2) / \epsilon_{2},(0.6,0.3) / \epsilon_{3},(0.5,0.2) / \epsilon_{4},(0.6,0.2) / \epsilon_{5},(0.6,0.3) / \epsilon_{6}\right\}$,

$\operatorname{med}_{\hat{\Gamma}}(\hat{\rho})=(0.55,0.2)$.

The med-level soft set is given as

$$
\begin{array}{r}
\mathcal{L}(\hat{\mathcal{F}} ; \text { med })=\left\{\left\{\ell_{2}, \ell_{3}, \ell_{4}\right\} / \epsilon_{1},\left\{\ell_{3}, \ell_{4}, \ell_{5}\right\} / \epsilon_{2},\left\{\ell_{1}, \ell_{2}, \ell_{3}\right\} / \epsilon_{3},\left\{\ell_{1}, \ell_{2}, \ell_{3}\right\} / \epsilon_{4},\left\{\ell_{1}, \ell_{2}, \ell_{4}, \ell_{5}\right\}\right. \\
\left./ \epsilon_{5},\left\{\ell_{1}, \ell_{3}, \ell_{4}\right\} / \epsilon_{6},\right\},
\end{array}
$$

$\mathcal{L}(\hat{\rho}$, med $)=\left\{\epsilon_{2}, \epsilon_{3}, \epsilon_{4}\right\}$.

Table 3. Level soft set $\mathcal{L}(\hat{\Gamma} ;$ mid $)$

\begin{tabular}{ccccccc}
\hline$\hat{\mathcal{Y}}$ & $\boldsymbol{\epsilon}_{\mathbf{1}}$ & $\boldsymbol{\epsilon}_{\mathbf{2}}$ & $\boldsymbol{\epsilon}_{\mathbf{3}}$ & $\boldsymbol{\epsilon}_{\mathbf{4}}$ & $\boldsymbol{\epsilon}_{\mathbf{5}}$ & $\boldsymbol{\epsilon}_{\mathbf{6}}$ \\
\hline$\ell_{1}$ & 1 & 0 & 0 & 1 & 1 & 0 \\
$\ell_{2}$ & 0 & 0 & 0 & 0 & 1 & 0 \\
$\ell_{3}$ & 0 & 1 & 1 & 1 & 0 & 1 \\
$\ell_{4}$ & 1 & 1 & 1 & 0 & 1 & 1 \\
$\ell_{5}$ & 0 & 0 & 1 & 0 & 1 & 0 \\
\hline$\hat{\rho}$ & 0 & 1 & 0 & 1 & 1 & 0 \\
\hline
\end{tabular}


Table 4. Level soft set $\mathcal{L}(\hat{\Gamma} ; t b)$

\begin{tabular}{ccccccc}
\hline$\hat{\mathcal{Y}}$ & $\boldsymbol{\epsilon}_{\mathbf{1}}$ & $\boldsymbol{\epsilon}_{\mathbf{2}}$ & $\boldsymbol{\epsilon}_{\mathbf{3}}$ & $\boldsymbol{\epsilon}_{\mathbf{4}}$ & $\boldsymbol{\epsilon}_{\mathbf{5}}$ & $\boldsymbol{\epsilon}_{\mathbf{6}}$ \\
\hline$\ell_{1}$ & 1 & 0 & 0 & 0 & 0 & 0 \\
$\ell_{2}$ & 0 & 0 & 0 & 0 & 0 & 0 \\
$\ell_{3}$ & 0 & 0 & 0 & 1 & 0 & 1 \\
$\ell_{4}$ & 1 & 1 & 0 & 0 & 0 & 0 \\
$\ell_{5}$ & 0 & 0 & 1 & 0 & 1 & 0 \\
\hline$\hat{\rho}$ & 0 & 0 & 0 & 0 & 0 & 0 \\
\hline
\end{tabular}

Table 5. Level soft set $\mathcal{L}(\hat{\Gamma} ; t t)$

\begin{tabular}{ccccccc}
\hline$\hat{\mathcal{Y}}$ & $\epsilon_{1}$ & $\epsilon_{2}$ & $\epsilon_{3}$ & $\epsilon_{4}$ & $\epsilon_{5}$ & $\epsilon_{6}$ \\
\hline$\ell_{1}$ & 1 & 0 & 0 & 1 & 0 & 1 \\
$\ell_{2}$ & 0 & 0 & 0 & 0 & 0 & 0 \\
$\ell_{3}$ & 0 & 0 & 0 & 1 & 0 & 1 \\
$\ell_{4}$ & 1 & 1 & 0 & 0 & 1 & 0 \\
$\ell_{5}$ & 0 & 0 & 1 & 0 & 1 & 0 \\
\hline$\hat{\rho}$ & 0 & 1 & 0 & 0 & 0 & 0 \\
\hline
\end{tabular}

Table 6. Level soft set $\mathcal{L}(\hat{\Gamma} ; b b)$

\begin{tabular}{ccccccc}
\hline$\hat{\mathcal{Y}}$ & $\boldsymbol{\epsilon}_{\mathbf{1}}$ & $\boldsymbol{\epsilon}_{\mathbf{2}}$ & $\boldsymbol{\epsilon}_{\mathbf{3}}$ & $\boldsymbol{\epsilon}_{\mathbf{4}}$ & $\boldsymbol{\epsilon}_{\mathbf{5}}$ & $\boldsymbol{\epsilon}_{\mathbf{6}}$ \\
\hline$\ell_{1}$ & 1 & 0 & 0 & 0 & 1 & 0 \\
$\ell_{2}$ & 0 & 0 & 0 & 1 & 0 & 0 \\
$\ell_{3}$ & 0 & 1 & 0 & 1 & 0 & 1 \\
$\ell_{4}$ & 1 & 1 & 1 & 0 & 0 & 0 \\
$\ell_{5}$ & 0 & 0 & 1 & 0 & 1 & 0 \\
\hline$\hat{\rho}$ & 0 & 0 & 0 & 0 & 1 & 0 \\
\hline
\end{tabular}

Table 7. Level soft set $\mathcal{L}(\hat{\Gamma} ;$ med $)$

\begin{tabular}{ccccccc}
\hline$\hat{\mathcal{Y}}$ & $\epsilon_{\mathbf{1}}$ & $\boldsymbol{\epsilon}_{\mathbf{2}}$ & $\boldsymbol{\epsilon}_{\mathbf{3}}$ & $\boldsymbol{\epsilon}_{\mathbf{4}}$ & $\boldsymbol{\epsilon}_{\mathbf{5}}$ & $\boldsymbol{\epsilon}_{\mathbf{6}}$ \\
\hline$\ell_{1}$ & 1 & 0 & 0 & 1 & 1 & 1 \\
$\ell_{2}$ & 0 & 1 & 0 & 1 & 1 & 0 \\
$\ell_{3}$ & 0 & 1 & 1 & 1 & 0 & 1 \\
$\ell_{4}$ & 1 & 1 & 1 & 0 & 1 & 1 \\
$\ell_{5}$ & 0 & 0 & 1 & 0 & 1 & 0 \\
\hline$\hat{\rho}$ & 0 & 1 & 0 & 1 & 1 & 0 \\
\hline
\end{tabular}

Remark 1. In GIFSS we do not define bottom-top ( $b t$ ) threshold function because in this function we consider a lower bound of membership function and an upper bound of non-membership function and that's why it is dispensable and we always get a unit table. For example, if we consider the GIFSS use in Example 1, then

$$
\begin{aligned}
& b t_{\hat{\Gamma}}=\left\{(0.5,0.4) / \epsilon_{1},(0.6,0.3) / \epsilon_{2},(0.4,0.5) / \epsilon_{3},(0.2,0.6) / \epsilon_{4},(0.2,0.5) / \epsilon_{5},(0.3,0.5) / \epsilon_{6}\right\} \\
& b t_{\hat{\Gamma}}(\hat{\rho})=(0.4,0.4) .
\end{aligned}
$$

The level soft set related to bottom-top threshold function is defined in Table 8. 
Table 8. Level soft set $\mathcal{L}(\hat{\Gamma} ; b t)$

\begin{tabular}{ccccccc}
\hline$\hat{\mathcal{Y}}$ & $\boldsymbol{\epsilon}_{\mathbf{1}}$ & $\boldsymbol{\epsilon}_{\mathbf{2}}$ & $\boldsymbol{\epsilon}_{\mathbf{3}}$ & $\boldsymbol{\epsilon}_{\mathbf{4}}$ & $\boldsymbol{\epsilon}_{\mathbf{5}}$ & $\boldsymbol{\epsilon}_{\mathbf{6}}$ \\
\hline$\ell_{1}$ & 1 & 1 & 1 & 1 & 1 & 1 \\
$\ell_{2}$ & 1 & 1 & 1 & 1 & 1 & 1 \\
$\ell_{3}$ & 1 & 1 & 1 & 1 & 1 & 1 \\
$\ell_{4}$ & 1 & 1 & 1 & 1 & 1 & 1 \\
$\ell_{5}$ & 1 & 1 & 1 & 1 & 1 & 1 \\
\hline$\hat{\rho}$ & 1 & 1 & 1 & 1 & 1 & 1 \\
\hline
\end{tabular}

Theorem 3. Let $\hat{\Gamma}=(\hat{\mathcal{F}}, \hat{\mathcal{A}}, \hat{\rho})$ be a GIFSS over $\hat{\mathcal{Y}}$ and $\hat{\mathcal{A}} \subseteq \hat{P}$ a parametric set. Then we have the following characteristics:

1. $\mathcal{L}(\hat{\Gamma} ; t b) \subseteq \mathcal{L}(\hat{\Gamma} ;$ mid $)$

2. $\quad \mathcal{L}(\hat{\Gamma} ; t b) \subseteq \mathcal{L}(\hat{\Gamma} ; t t) ;$

3. $\quad \mathcal{L}(\hat{\Gamma} ; t b) \subseteq \mathcal{L}(\hat{\Gamma} ; b b)$.

Proof. In the following we prove only first part, remaining parts prove similarly.

Let $\mathcal{L}(\hat{\Gamma} ; t b)=\left(\hat{\mathcal{F}}_{t b}, \hat{\mathcal{A}}, \hat{\rho}_{t b}\right)$ and $\mathcal{L}(\hat{\Gamma} ;$ mid $)=\left(\hat{\mathcal{F}}_{\text {mid }}, \hat{\mathcal{A}}, \hat{\rho}_{\text {mid }}\right)$. To complete the proof, we need to show $\left(\hat{\mathcal{F}}_{t b}, \hat{\mathcal{A}}\right) \subseteq\left(\hat{\mathcal{F}}_{\text {mid }}, \hat{\mathcal{A}}\right)$ and $\mathcal{L}(\hat{\rho} ; t b) \subseteq \mathcal{L}(\hat{\rho} ;$ mid $)$. To show $\left(\hat{\mathcal{F}}_{t b}, \hat{\mathcal{A}}\right) \subseteq\left(\hat{\mathcal{F}}_{\text {mid }}, \hat{\mathcal{A}}\right)$, we have to show $\hat{\mathcal{F}}_{t b}(\epsilon) \subseteq \hat{\mathcal{F}}_{\text {mid }}(\epsilon)$ for all $\epsilon \in \hat{\mathcal{A}}$. Let $\hat{\mathcal{F}}_{t b}(\epsilon)=\left\{\ell \in \hat{\mathcal{Y}} \mid \xi_{\hat{\mathcal{F}}(\epsilon)}(\ell) \geq k_{t b_{\hat{\Gamma}}}(\epsilon)\right.$ and $\left.\vartheta_{\hat{\mathcal{F}}(\epsilon)}(\ell) \leq l_{t b_{\hat{\Gamma}}}(\epsilon)\right\}$ and $\hat{\mathcal{F}}_{\text {mid }}(\epsilon)=\left\{\ell \in \hat{\mathcal{Y}} \mid \xi_{\hat{\mathcal{F}}(\epsilon)}(\ell) \geq k_{\text {mid }_{\hat{\Gamma}}}(\epsilon)\right.$ and $\left.\vartheta_{\hat{\mathcal{F}}(\epsilon)}(\ell) \leq l_{\text {mid }}(\epsilon)\right\}$. Since

$$
\begin{gathered}
k_{t b_{\hat{\Gamma}}}(\epsilon)=\max _{\ell \in \hat{\mathcal{Y}}} \xi_{\hat{\mathcal{F}}(\epsilon)}(\ell) \geq \frac{1}{|\hat{\mathcal{Y}}|} \sum_{\ell \in \hat{\mathcal{Y}}} \xi_{\hat{\mathcal{F}}(\epsilon)}(\ell)=k_{\operatorname{mid}_{\hat{\Gamma}}}(\epsilon) k_{t b_{\hat{\Gamma}}}(\epsilon) \geq k_{\operatorname{mid}_{\hat{\Gamma}}}(\epsilon), \\
l_{t b_{\hat{\Gamma}}}(\epsilon)=\min _{\ell \in \hat{\mathcal{Y}} \vartheta_{\hat{\mathcal{F}}(\epsilon)}(\ell)} \leq \frac{1}{|\hat{\mathcal{Y}}|} \sum_{\ell \in \hat{\mathcal{Y}}} \vartheta_{\hat{\mathcal{F}}(\epsilon)}(\ell)=l_{\operatorname{mid}_{\hat{\Gamma}}}(\epsilon) \\
l_{t b_{\hat{\Gamma}}}(\epsilon) \leq l_{\operatorname{mid}_{\hat{\Gamma}}}(\epsilon) .
\end{gathered}
$$

If $\ell \in \hat{\mathcal{F}}_{t b}(\epsilon)$, then we have $\xi_{\hat{\mathcal{F}}(\epsilon)}(\ell) \geq k_{t b_{\hat{\Gamma}}}(\epsilon) \geq k_{m i d_{\hat{\Gamma}}}(\epsilon)$ and $\vartheta_{\hat{\mathcal{F}}(\epsilon)}(\ell) \leq l_{t b_{\hat{\Gamma}}}(\epsilon) \leq l_{m i d_{\hat{\Gamma}}}(\epsilon)$, and hence $\ell \in \hat{\mathcal{F}}_{\text {mid }}(\epsilon)$.

Now, let $\mathcal{L}(\hat{\rho} ; t b)=\left\{\epsilon \in \hat{\mathcal{A}} \mid \xi_{\hat{\rho}(\epsilon)} \geq u_{t b}\right.$ and $\left.\vartheta_{\hat{\rho}(\epsilon)} \leq v_{t b}\right\}$ and $\mathcal{L}(\hat{\rho} ;$ mid $)=\left\{\epsilon \in \hat{\mathcal{A}} \mid \xi_{\hat{\rho}(\epsilon)} \geq\right.$ $u_{\text {mid }}$ and $\left.\vartheta_{\hat{\rho}(\epsilon)} \leq v_{\text {mid }}\right\}$ for all $\epsilon \in \hat{\mathcal{A}}$. Since

$$
\begin{aligned}
u_{t b_{\hat{\Gamma}}}=\max _{\epsilon \in \hat{\mathcal{A}}} \xi_{\hat{\rho}(\epsilon)} & \geq \frac{1}{|\hat{\mathcal{A}}|} \sum_{\epsilon \in \hat{\mathcal{A}}} \xi_{\hat{\rho}(\epsilon)}=u_{m i d_{\hat{\Gamma}}} \\
u_{t b_{\hat{\Gamma}}} & \geq u_{m i d_{\hat{\Gamma}^{\prime}}} \\
v_{t b_{\hat{\Gamma}}}=\min _{\epsilon \in \hat{\mathcal{A}}} \vartheta_{\hat{\rho}(\epsilon)} & \leq \frac{1}{|\hat{\mathcal{A}}|} \sum_{\epsilon \in \hat{\mathcal{A}}} \vartheta_{\hat{\rho}(\epsilon)}=v_{m i d_{\hat{\Gamma}}} \\
v_{t b_{\hat{\Gamma}}} & \leq v_{\operatorname{mid}_{\hat{\Gamma}}} .
\end{aligned}
$$

If $\epsilon \in \mathcal{L}(\hat{\rho} ; t b)$, then $\xi_{\hat{\rho}(\epsilon)} \geq u_{t b} \geq u_{m i d_{\hat{\Gamma}}}$ and $\vartheta_{\hat{\rho}(\epsilon)} \leq v_{t b} \leq v_{\text {mid }_{\hat{\Gamma}}}$. Hence $\epsilon \in \mathcal{L}(\hat{\rho} ;$ mid $)$ and we are done. 


\section{Applications of the GIFSS Model Based on Weighted Soft Discernibility Matrix}

The idea of GIFSS is very encouraging in decision-making since it considers how to capitalize an additional intuitionistic fuzzy input from the director to minimize any possible perversion in the data provided by evaluating specialists. Also, in our daily life decision making problems, different attributes are not of equal importance. Some are more important than others; therefore, the decision maker assigns different values (weights) to different attributes and imposed different thresholds functions when we need to put restriction on membership function and non-membership function. First, Skowron and Rauszer [43] initiated the concept of discernibility matrix and extensively used in rough sets to solve attribute reduction and the influence of it are significant and easy to understand. In this paper, we use an adjustable perspective to GIFSS and get level soft sets. Then each GIFSS can be seen as the level soft set and composed a crisp soft set, therefore, for solving decision making problems we apply $\mathcal{S D} \mathcal{M}$. The soft equivalence relation in soft sets is a bridge among soft sets and rough sets which is introduced by Ali [44].

Definition 15. The accuracy weighted choice value of an alternative $\ell_{i} \in \hat{\mathcal{Y}}$ is $f_{i}$ given by $f_{i}=\sum e_{i j}$, where $e_{i j}=$ $\hat{\omega}_{i j} \times \ell_{i j}$, where $\hat{\omega}_{i j}$ are the accuracy weights calculated from PIFS using accuracy function defined as $\hat{\omega}_{i j}=\xi_{j}+\vartheta_{j}$.

Definition 16. The expectation score weighted choice value of an alternative $\ell_{i} \in \hat{\mathcal{Y}}$ is $f_{i}$ given by $f_{i}=\sum e_{i j}$, where $e_{i j}=\hat{\omega}_{i j} \times \ell_{i j}$, where $\hat{\omega}_{i j}$ are the expectation score weights calculated from PIFS using expectation score function defined as $\hat{\omega}_{i j}=\left(\xi_{j}-\vartheta_{j}+1\right) / 2$.

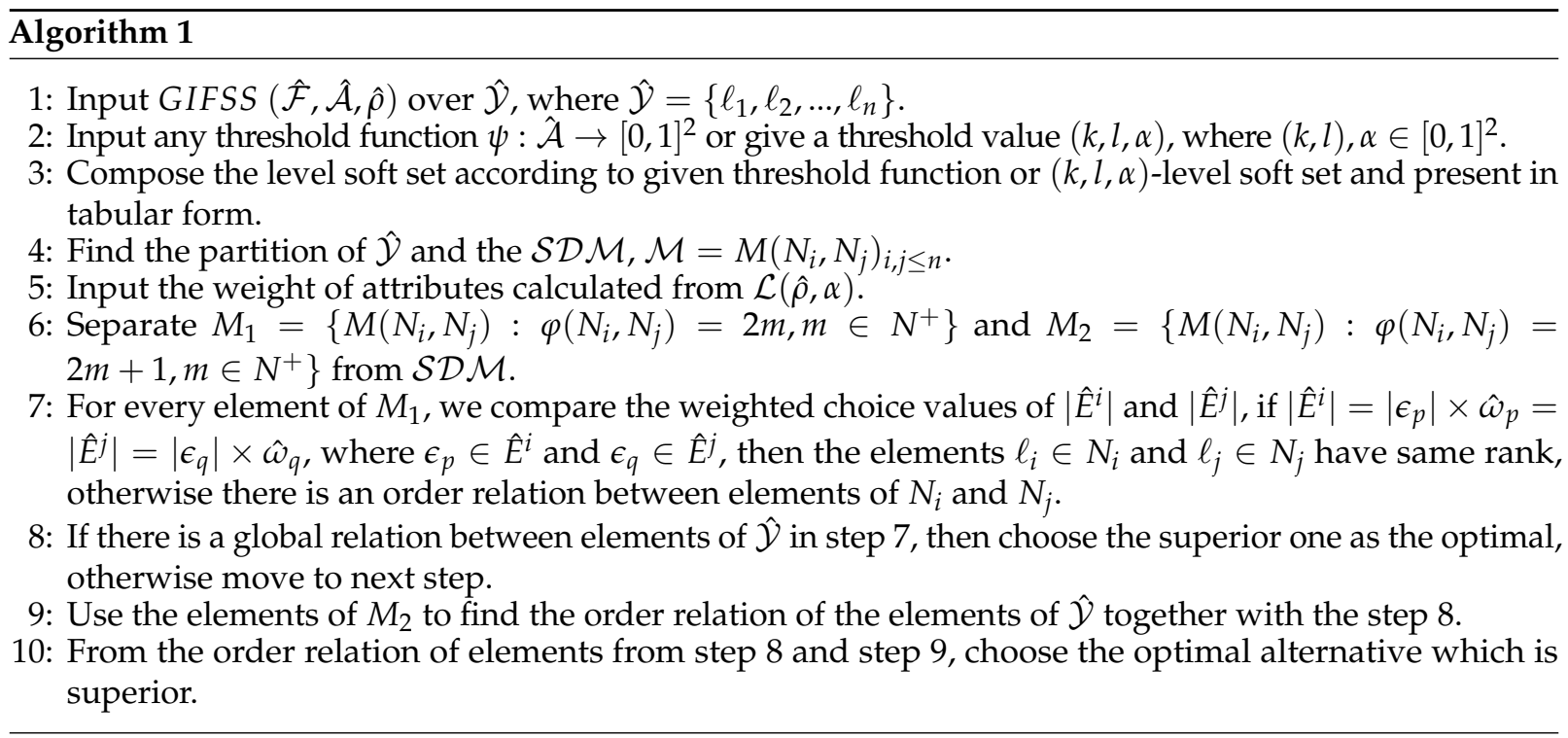

From Reference [44], we have no concern with the values of decision parameter $\mathcal{D}=\sum \ell_{i j}$ but actually we are interested on investigation its classification ability. Only from the parameters value in the set of $S D P s$, classification ability of decision parameter is determined. Thus, we only need to compare the values of the decision parameter restricted within every set of SDPs.

Example 3. Given a GIFSS $(\hat{\mathcal{F}}, \hat{\mathcal{A}}, \hat{\rho})$ as in Example 1, find the order relation among all the alternatives of GIFSS using Algoritm 1. We use the med-level threshold function and compute the level soft set $\mathcal{L}(\hat{\Gamma} ;$ med $)$ as in Table 7. From Table 7 , the parameters $\epsilon_{2}, \epsilon_{4}, \epsilon_{5}$ have value 1 , therefore, the weighted value of $\epsilon_{2}, \epsilon_{4}$ and $\epsilon_{5}$ are 1 (say) and the parameters $\epsilon_{1}, \epsilon_{3}, \epsilon_{6}$ have value 0 , therefore, the weight value of $\epsilon_{1}, \epsilon_{3}$ and $\epsilon_{6}$ are 0.7 . 
From Table 7 , we can obtained the partition of $\hat{\mathcal{Y}}$ is

$$
\left\{\left\{\ell_{1}\right\},\left\{\ell_{2}\right\},\left\{\ell_{3}\right\},\left\{\ell_{4}\right\},\left\{\ell_{5}\right\}\right\} \text {. }
$$

We will denote $N_{1}=\left\{\ell_{1}\right\}, N_{2}=\left\{\ell_{2}\right\}, N_{3}=\left\{\ell_{3}\right\}, N_{4}=\left\{\ell_{4}\right\}$ and $N_{5}=\left\{\ell_{5}\right\}$ and also constructed $\mathcal{S D} \mathcal{M}$ in Table 9.

Table 9. The $\mathcal{W} \mathcal{S D} \mathcal{M}$ of Example 3.

\begin{tabular}{cccccc}
\hline$N_{i}$ & $N_{1}$ & $N_{2}$ & $N_{3}$ & $N_{4}$ & $N_{5}$ \\
\hline$N_{1}$ & $\varnothing$ & & & \\
$N_{2}$ & $\left\{\epsilon_{1}^{1 * 0.7}, \epsilon_{6}^{1 * 0.7}, \epsilon_{2}^{2 * 1}\right\}$ & $\varnothing$ & & \\
$N_{3}$ & $\left\{\epsilon_{1}^{1 * 0.7}, \epsilon_{5}^{1 * 1}, \epsilon_{2}^{3 * 1}, \epsilon_{3}^{3 * 0.7}\right\}$ & $\left\{\epsilon_{5}^{2 * 1}, \epsilon_{3}^{3 * 0.7}, \epsilon_{6}^{3 * 0.7}\right\}$ & $\varnothing$ & \\
$N_{4}$ & $\left\{\epsilon_{4}^{1+1}, \epsilon_{2}^{* 1}, \epsilon_{3}^{4 * 0.7}\right\}$ & $\left\{\epsilon_{4}^{2 * 1}, \epsilon_{6}^{4 * 0.7}, \epsilon_{1}^{4 * 0.7}, \epsilon_{3}^{4 * 0.7}\right\}$ & $\left\{\epsilon_{4}^{3 * 1}, \epsilon_{1}^{4 * 0.7}, \epsilon_{5}^{4 * 1}\right\}$ & $\varnothing$ \\
$N_{5}$ & $\left\{\epsilon_{1}^{1 * 0.7}, \epsilon_{4}^{1 * 1}, \epsilon_{6}^{1 * 0.7}, \epsilon_{3}^{5 * 0.7}\right\}$ & $\left\{\epsilon_{2}^{2 * 1}, \epsilon_{4}^{2 * 1}, \epsilon_{3}^{5 * 0.7}\right\}$ & $\left\{\epsilon_{2}^{3 * 1}, \epsilon_{4}^{3 * 1}, \epsilon_{6}^{3 * 0.7}, \epsilon_{5}^{5 * 0.7}\right\}\left\{\epsilon_{1}^{4 * 0.7}, \epsilon_{2}^{* * 1}, \epsilon_{6}^{4 * 0.7}\right\}$ & $\varnothing$ \\
\hline
\end{tabular}

From Table 9, we have

$$
\begin{aligned}
& M_{1}=\left\{M\left(N_{1}, N_{3}\right), M\left(N_{1}, N_{5}\right), M\left(N_{2}, N_{4}\right), M\left(N_{3}, N_{5}\right)\right\} . \\
& M_{2}=\left\{M\left(N_{1}, N_{2}\right), M\left(N_{1}, N_{4}\right), M\left(N_{2}, N_{3}\right), M\left(N_{2}, N_{5}\right), M\left(N_{3}, N_{4}\right), M\left(N_{4}, N_{5}\right)\right\} .
\end{aligned}
$$

From $M_{1}$, we know that in $M\left(N_{1}, N_{3}\right),\left|\hat{E}^{1}\right|=\left|\epsilon_{1}\right| \times 0.7+\left|\epsilon_{5}\right| \times 1=1.7$ and $\left|\hat{E}^{3}\right|=\left|\epsilon_{2}\right| \times 1+\left|\epsilon_{3}\right| \times 0.7=$ 1.7, so the alternatives in $\hat{E}^{1}$ and $\hat{E}^{3}$ have same rank, in other words $\ell_{1}$ and $\ell_{3}$ are in same decision class. Similarly, in $M\left(N_{1}, N_{5}\right),\left|\hat{E}^{1}\right|>\left|\hat{E}^{5}\right|$, the alternative in $N_{1}$ is superior to the alternative in $N_{5}$, that is, $\ell_{1}$ is superior to $\ell_{5}$. From $M\left(N_{1}, N_{3}\right)$, we have $\ell_{1}, \ell_{3}$ are superior to $\ell_{5}$. From $M\left(N_{1}, N_{2}\right),\left|\hat{E}^{1}\right|>\left|\hat{E}^{2}\right|$, the alternative in $N_{1}$ is superior to the alternative in $N_{2}$, that is, $\ell_{1}$ is superior to $\ell_{2}$. From $M\left(N_{1}, N_{3}\right)$, we have $\ell_{1}$ and $\ell_{3}$ are superior to $\ell_{2}$. From $M\left(N_{1}, N_{4}\right),\left|\hat{E}^{4}\right|>\left|\hat{E}^{1}\right|$, the alternative in $N_{4}$ is superior to the alternative in $N_{1}$, that is, $\ell_{4}$ is superior to $\ell_{1}$, hence $\ell_{4}$ is superior to $\ell_{3}$. From $M\left(N_{2}, N_{5}\right),\left|\hat{E}^{2}\right|>\left|\hat{E}^{5}\right|$, the alternative in $N_{2}$ is superior to the alternative in $N_{5}$, that is, $\ell_{2}$ is superior to $\ell_{5}$.

Combining the above results, we have $\ell_{4} \succ\left\{\ell_{1}, \ell_{3}\right\} \succ \ell_{2} \succ \ell_{5}$, so an order relation among all the alternatives is obtained. And the best alternative is $\ell_{4}$.

We have seen that when we solve decision making problem using $\mathcal{S D} \mathcal{M}$, some attributes will be erased unintentionally. Thus, by constructing $\mathcal{S D M}$ for a soft set some attributes which have no impact on the final conclusion will be erased.

\section{Algorithm 2}

Input: The $\operatorname{GIFSS}(\hat{\mathcal{F}}, \hat{\mathcal{A}}, \hat{\rho})$ over $\hat{\mathcal{Y}}$, where $\hat{\mathcal{Y}}=\left\{\ell_{1}, \ell_{2}, \ldots, \ell_{n}\right\}$.

Output: The order relation of all the alternatives.

1: Input the threshold function $\psi: \hat{\mathcal{A}} \rightarrow[0,1]^{2}$ or give a threshold value $(k, l)$, where $(k, l) \in[0,1]^{2}$.

2: Compute the level soft set of BIFSS accordingly and present it in tabular form.

3: Compute the partition of $\hat{\mathcal{Y}}$ and $\mathcal{S D} \mathcal{M}, \mathcal{M}=M\left(N_{i}, N_{j}\right)_{i, j \leq n}$.

4: Find the accuracy weights or expectation score weights of attributes from PIFS.

5: Input the weights calculated in step 4 of attributes into the $\mathcal{S D} \mathcal{M}$.

6: Compare the weighted choice values of $M\left(N_{i}, N_{j}\right)$ in the similar way as in Algorithm 1.

7: Output the order relation among all the alternatives.

Example 4. For the GIFSS define in Table 1 , the $\left(k_{\text {med }}, l_{\text {med }}\right)$-level soft set have calculated in Table 7 , and the partition $M_{1}$ and $M_{2}$ are calculated in Example 3. The accuracy weights of each parameter is given as follows: 
$\hat{\omega}_{1}=0.6, \hat{\omega}_{2}=1, \hat{\omega}_{3}=0.8, \hat{\omega}_{4}=0.8, \hat{\omega}_{5}=0.8$ and $\hat{\omega}_{6}=0.8$. Then we have a accuracy $\mathcal{W} \mathcal{S} \mathcal{D} \mathcal{M}$ with the tabular representation shown in Table 10.

Table 10. The accuracy $\mathcal{W} \mathcal{S} \mathcal{D} \mathcal{M}$ of Example 4.

\begin{tabular}{cccccc}
\hline$N_{i}$ & $N_{\mathbf{1}}$ & $N_{\mathbf{2}}$ & $N_{\mathbf{3}}$ & $N_{\mathbf{4}}$ & $N_{\mathbf{5}}$ \\
\hline$N_{1}$ & $\varnothing$ & & & & \\
$N_{2}$ & $\left\{\epsilon_{1}^{1 * 0.6}, \epsilon_{6}^{1 * 0.8}, \epsilon_{2}^{2 * 1}\right\}$ & $\varnothing$ & & \\
$N_{3}$ & $\left\{\epsilon_{1}^{1 * 0.6}, \epsilon_{5}^{1 * 0.8}, \epsilon_{2}^{3 * 1}, \epsilon_{3}^{3 * 0.8}\right\}$ & $\left\{\epsilon_{5}^{2 * 0.8}, \epsilon_{3}^{3 * 0.8}, \epsilon_{6}^{3 * 0.8}\right\}$ & $\varnothing$ & \\
$N_{4}$ & $\left\{\epsilon_{4}^{1 * 0.8}, \epsilon_{2}^{4 * 1}, \epsilon_{3}^{4 * 0.8}\right\}$ & $\left\{\epsilon_{4}^{2 * 0.8}, \epsilon_{6}^{4 * 0.8}, \epsilon_{1}^{4 * 0.6}, \epsilon_{3}^{4 * 0.8}\right\}$ & $\left\{\epsilon_{4}^{3 * 0.8}, \epsilon_{1}^{4 * 0.6}, \epsilon_{5}^{4 * 0.8}\right\}$ & $\varnothing$ \\
$N_{5}$ & $\left\{\epsilon_{1}^{1 * 0.6}, \epsilon_{4}^{1 * 0.8}, \epsilon_{6}^{1 * 0.8}, \epsilon_{3}^{5 * 0.8}\right\}$ & $\left\{\epsilon_{2}^{2 * 1}, \epsilon_{4}^{2 * 0.8}, \epsilon_{3}^{5 * 0.8}\right\}$ & $\left\{\epsilon_{2}^{3 * 1}, \epsilon_{4}^{3 * 0.8}, \epsilon_{6}^{3 * 0.8}, \epsilon_{5}^{5 * 0.8}\right\}$ & $\left\{\epsilon_{1}^{4 * 0.6}, \epsilon_{2}^{4 * 1}, \epsilon_{6}^{4 * 0.8}\right\}$ & $\varnothing$ \\
\hline
\end{tabular}

From Table 10, we know that in $M\left(N_{1}, N_{3}\right),\left|\hat{E}^{1}\right|=\left|\epsilon_{1}\right| \times 0.6+\left|\epsilon_{5}\right| \times 0.8=1.4$ and $\left|\hat{E}^{3}\right|=\left|\epsilon_{2}\right| \times 1+$ $\left|\epsilon_{5}\right| \times 0.8=1.8$, thus the alternative in $N_{3}$ is superior to the alternative in $N_{1}$, that is, $\ell_{3}$ is superior to $\ell_{1}$. Similarly, we can obtained the order relation among the all alternatives is $\ell_{4} \succ \ell_{3} \succ \ell_{1} \succ \ell_{2} \succ \ell_{5}$. Hence the optimal alternative is $\ell_{4}$.

\section{Case Study for Selecting Candidates for Ph.D. Scholarships}

A mathematics department of university $Y$ has three scholarships for a doctoral degree. Many students apply for a scholarship but due to initial conditions on CGPA (cumulative grade points average), only seven students are short listed for further evaluation. Let $\hat{\mathcal{Y}}=\left\{\ell_{1}, \ell_{2}, \ldots, \ell_{7}\right\}$ represent the alternatives (students) and $\hat{P}=\left\{\epsilon_{1}, \epsilon_{2}, \ldots, \epsilon_{6}\right\}$ are the attributes (criteria), where each $\epsilon_{i}$ stands for "CGPA", "no. of research papers", "research quality", "research proposal", "personal statement" and "interview". For selection, the vice chancellor of the university set up a committee of experts to make an evaluation on the basis of given criteria (attributes). The committee evaluates students and given their evaluation in the form of $B I F S S$ and vice chancellor scrutinizes the general quality of evaluation made by an expert group and gives his view in the form of PIFS, which completes the construction of GIFSS, $\hat{\Gamma}=(\hat{\mathcal{F}}, \hat{\mathcal{A}}, \hat{\rho})$. The tabular representation of GIFSS is given in Table 11.

Table 11. The $\operatorname{GIFSS}(\hat{\mathcal{F}}, \hat{\mathcal{A}}, \hat{\rho})$.

\begin{tabular}{ccccccc}
\hline$\hat{\mathcal{Y}}$ & $\epsilon_{1}$ & $\epsilon_{2}$ & $\epsilon_{3}$ & $\epsilon_{4}$ & $\epsilon_{5}$ & $\epsilon_{6}$ \\
\hline$\ell_{1}$ & $(0.6,0.3)$ & $(0.7,0.3)$ & $(0.4,0.4)$ & $(0.3,0.7)$ & $(0.8,0.2)$ & $(0.4,0.6)$ \\
$\ell_{2}$ & $(0.3,0.5)$ & $(0.4,0.5)$ & $(0.6,0.3)$ & $(0.5,0.4)$ & $(0.4,0.4)$ & $(0.6,0.4)$ \\
$\ell_{3}$ & $(0.5,0.4)$ & $(0.8,0.2)$ & $(0.5,0.4)$ & $(0.8,0.1)$ & $(0.7,0.3)$ & $(0.7,0.3)$ \\
$\ell_{4}$ & $(0.7,0.2)$ & $(0.5,0.4)$ & $(0.4,0.5)$ & $(0.9,0.1)$ & $(0.3,0.5)$ & $(0.3,0.5)$ \\
$\ell_{5}$ & $(0.8,0.2)$ & $(0.6,0.3)$ & $(0.8,0.2)$ & $(0.2,0.7)$ & $(0.5,0.3)$ & $(0.5,0.4)$ \\
$\ell_{6}$ & $(0.4,0.5)$ & $(0.7,0.2)$ & $(0.7,0.2)$ & $(0.4,0.4)$ & $(0.4,0.5)$ & $(0.4,0.3)$ \\
$\ell_{7}$ & $(0.5,0.5)$ & $(0.3,0.6)$ & $(0.3,0.6)$ & $(0.4,0.5)$ & $(0.6,0.4)$ & $(0.6,0.3)$ \\
\hline$\hat{\rho}$ & $(0.7,0.3)$ & $(0.6,0.2)$ & $(0.3,0.4)$ & $(0.5,0.4)$ & $(0.3,0.3)$ & $(0.7,0.1)$ \\
\hline
\end{tabular}

We use med-level threshold function for BIFSS and the med-level soft set with its tabular representation (Table 12) is given as follows

$$
\operatorname{med}_{\hat{\Gamma}}=\left\{(0.5,0.4) / \epsilon_{1},(0.6,0.3) / \epsilon_{2},(0.5,0.4) / \epsilon_{3},(0.4,0.4) / \epsilon_{4},(0.5,0.4) / \epsilon_{5},(0.5,0.4) / \epsilon_{6}\right\} .
$$

From Table 12, we can obtained the partition of $\hat{\mathcal{Y}}$ is $N_{1}=\left\{\ell_{1}\right\}, N_{2}=\left\{\ell_{2}\right\}, N_{3}=\left\{\ell_{3}\right\}, N_{4}=\left\{\ell_{4}\right\}$, $N_{5}=\left\{\ell_{5}\right\}, N_{6}=\left\{\ell_{6}\right\}$ and $N_{7}=\left\{\ell_{7}\right\}$. We find the expectation score weights from PIFS, which are 
$\hat{\omega}_{1}=0.7, \hat{\omega}_{2}=0.7, \hat{\omega}_{3}=0.45, \hat{\omega}_{4}=0.55, \hat{\omega}_{5}=0.5$ and $\hat{\omega}_{6}=0.8$. From partition, we constructed $\mathcal{W} \mathcal{S} \mathcal{D}$ which is given in Table 13.

Table 12. Level Soft Set $\mathcal{L}(\hat{\Gamma} ;$ med $)$.

\begin{tabular}{ccccccc}
\hline$\hat{\mathcal{Y}}$ & $\boldsymbol{\epsilon}_{\mathbf{1}}$ & $\boldsymbol{\epsilon}_{\mathbf{2}}$ & $\boldsymbol{\epsilon}_{\mathbf{3}}$ & $\boldsymbol{\epsilon}_{\mathbf{4}}$ & $\boldsymbol{\epsilon}_{\mathbf{5}}$ & $\boldsymbol{\epsilon}_{\mathbf{6}}$ \\
\hline$\ell_{1}$ & 1 & 1 & 0 & 0 & 1 & 0 \\
$\ell_{2}$ & 0 & 0 & 1 & 1 & 0 & 1 \\
$\ell_{3}$ & 1 & 1 & 1 & 1 & 1 & 1 \\
$\ell_{4}$ & 1 & 0 & 0 & 1 & 0 & 0 \\
$\ell_{5}$ & 1 & 1 & 1 & 0 & 1 & 1 \\
$\ell_{6}$ & 0 & 1 & 1 & 1 & 0 & 0 \\
$\ell_{7}$ & 0 & 0 & 0 & 0 & 1 & 1 \\
\hline
\end{tabular}

Table 13. The $\mathcal{W S D} \mathcal{M}$ of case study in Section 5.

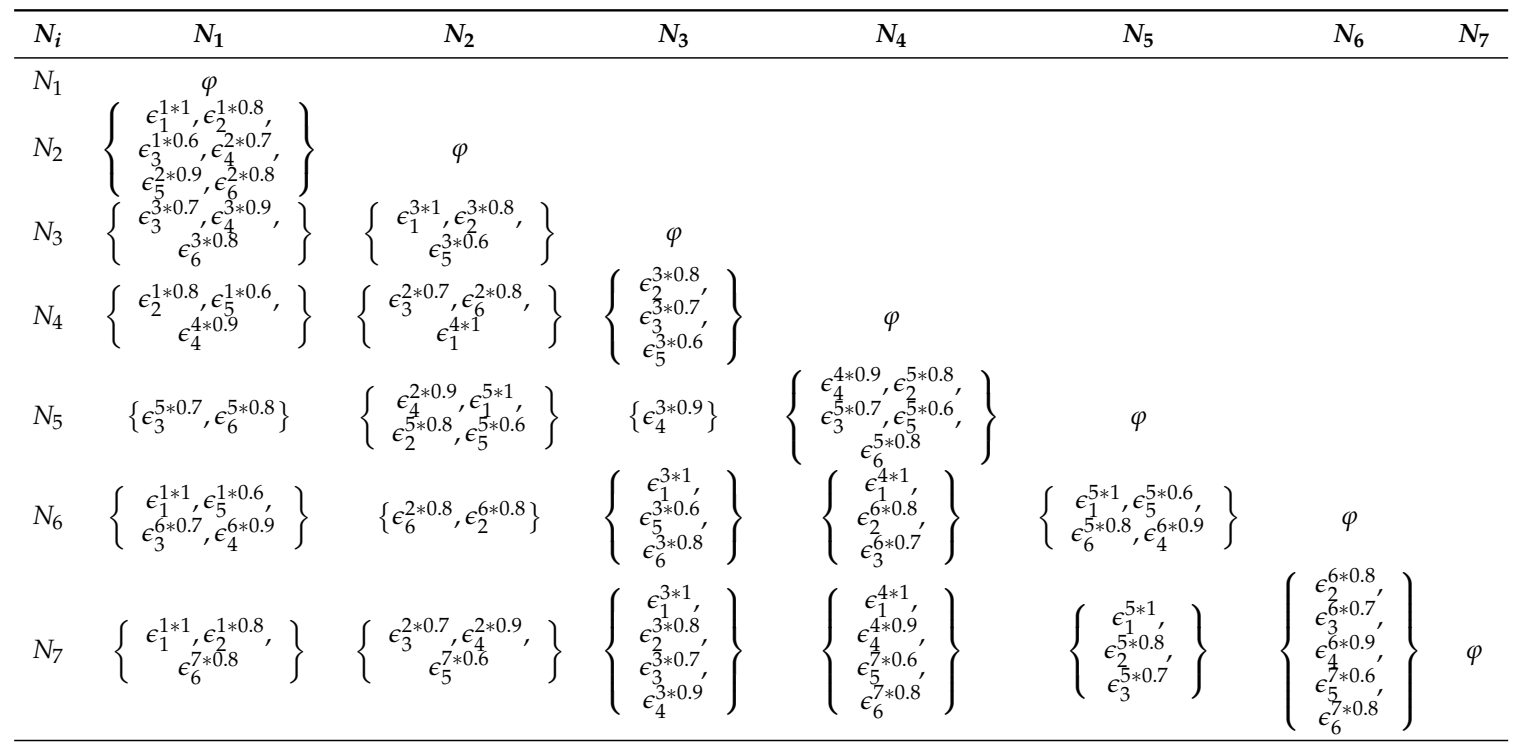

From Table 13, we have

$$
\begin{array}{r}
M_{1}=\left\{M\left(N_{1}, N_{2}\right), M\left(N_{1}, N_{5}\right), M\left(N_{1}, N_{6}\right), M\left(N_{2}, N_{5}\right), M\left(N_{2}, N_{6}\right), M\left(N_{3}, N_{7}\right), M\left(N_{4}, N_{7}\right), M\left(N_{5}, N_{6}\right)\right\} . \\
M_{2}=\left\{M\left(N_{1}, N_{3}\right), M\left(N_{1}, N_{4}\right), M\left(N_{1}, N_{7}\right), M\left(N_{2}, N_{3}\right), M\left(N_{2}, N_{4}\right), M\left(N_{2}, N_{7}\right), M\left(N_{3}, N_{4}\right), M\left(N_{2}, N_{4}\right),\right. \\
\left.M\left(N_{3}, N_{5}\right), M\left(N_{3}, N_{6}\right), M\left(N_{4}, N_{5}\right), M\left(N_{4}, N_{6}\right), M\left(N_{5}, N_{7}\right), M\left(N_{6}, N_{7}\right)\right\} .
\end{array}
$$

From $\mathcal{W S D} \mathcal{M}$, we know that in $M\left(N_{1}, N_{2}\right),\left|\hat{E}^{1}\right|=\left|\epsilon_{1}\right| \times 0.7+\left|\epsilon_{2}\right| \times 0.7+\left|\epsilon_{5}\right| \times 0.5=1.9$ and $\left|\hat{E}^{2}\right|=\left|\epsilon_{3}\right| \times 0.45+\left|\epsilon_{4}\right| \times 0.55+\left|\epsilon_{6}\right| \times 0.8=1.8$, thus we find that $N_{1}$ is superior to $N_{2}$, that is, $\ell_{1}$ is superior to $\ell_{2}$. Similarly, in $M\left(N_{1}, N_{3}\right),\left|\hat{E}^{3}\right|>\left|\hat{E}^{1}\right|$, the alternative in $N_{3}$ is superior to the alternative in $N_{1}$, that is, $\ell_{3}$ is superior to $\ell_{1}$. From $M\left(N_{1}, N_{4}\right),\left|\hat{E}^{1}\right|>\left|\hat{E}^{4}\right|$, the alternative in $N_{1}$ is superior to the alternative in $N_{4}$, that is, $\ell_{1}$ is superior to $\ell_{4}$. From $M\left(N_{1}, N_{5}\right)$, we have $\ell_{5}$ is superior to $\ell_{1}$. From $M\left(N_{1}, N_{6}\right)$, we have $\ell_{1}$ is superior to $\ell_{6}$. From $M\left(N_{1}, N_{7}\right)$, we have $\ell_{1}$ is superior to $\ell_{7}$. From $M\left(N_{2}, N_{4}\right)$, we have $\ell_{2}$ is superior to $\ell_{4}$. From $M\left(N_{4}, N_{6}\right)$, we have $\ell_{6}$ is superior to $\ell_{4}$. From $M\left(N_{2}, N_{6}\right)$, we have $\ell_{2}$ is superior to 
$\ell_{6}$. From $M\left(N_{6}, N_{7}\right)$, we have $\ell_{6}$ is superior to $\ell_{7}$. From $M\left(N_{4}, N_{7}\right)$, we have $\ell_{7}$ is superior to $\ell_{4}$. And from $M\left(N_{3}, N_{5}\right)$, we have $\ell_{3}$ is superior to $\ell_{5}$. Hence the order relation among the alternatives is

$$
\ell_{3} \succ \ell_{5} \succ \ell_{1} \succ \ell_{2} \succ \ell_{6} \succ \ell_{7} \succ \ell_{4}
$$

and $\ell_{3}, \ell_{5}$ and $\ell_{1}$ are the best candidates for scholarship.

\section{Comparison Analysis}

In this section, we compare our proposed algorithm with some related methods to indicate its advantages.

At first, we compare our method with the method proposed in Reference [22], where Agarwal define GIFSS and used it for different problems like medical diagnose and decision making but in Reference [23], Feng pointed out some problems in the proposed method and provided a counter example that shows that in calculating the NAE in the fifth step, the relation is not compatible with the $\otimes$ operation. Also in Reference [45], Khalil gives a counter example to some propositions of GIFSS. If we compare our method with the method proposed in Reference [23], we have seen that the we get same result as in Reference [23]. But it is not necessary that we get the same results because our method is different and helps in situations when all attributes are not of equal importance and we need to put some restrictions/boundaries on membership and non-membership functions. In our proposed method, we can find not only the best choice alternative, but also an order relation of all alternatives easily by scanning the $\mathcal{W S D M}$ at most one time. If we compare our method with the method proposed in Reference [40], we have seen that the previous method deals with the intuitionistic fuzzy soft sets and in his method he did not give any information on how to calculate the weights of parameters but in our proposed method we are working in GIFSS and give different criteria to find the weights of parameters by using parametric intuitionistic fuzzy soft set. We compare our method with the methods proposed in References [23,40,46-49] and results summarized in Table 14 taken from [23], we have seen that the order relation among all alternatives and optimal alternative is the same as we have. Only in Reference [46], we have seen that the $\ell_{3}$ is superior to $\ell_{1}$, but in remaining all methods $\ell_{1}$ is superior to $\ell_{3}$. Actually Lin use the measure $S_{\operatorname{Lin}}\left(\xi_{\hat{\mathcal{A}}}, \vartheta_{\hat{\mathcal{A}}}\right)=2 \xi_{\hat{\mathcal{A}}}+\vartheta_{\hat{\mathcal{A}}}-1$, which is increasing w.r.t the $\vartheta_{\hat{\mathcal{A}}}$. This is counter intuitive and might cause difficulties in some practical applications.

Table 14. Order of alternatives using different methods.

\begin{tabular}{cc}
\hline$\hat{\mathcal{Y}}$ & Order of the Alternatives \\
\hline$[23]$ & $\ell_{4} \succ \ell_{1} \succ \ell_{3} \succ \ell_{2} \succ \ell_{5}$ \\
{$[40]$} & $\ell_{4} \succ \ell_{1} \succ \ell_{3} \succ \ell_{2} \succ \ell_{5}$ \\
{$[46]$} & $\ell_{4} \succ \ell_{3} \succ \ell_{1} \succ \ell_{2} \succ \ell_{5}$ \\
{$[47]$} & $\ell_{4} \succ \ell_{1} \succ \ell_{3} \succ \ell_{2} \succ \ell_{5}$ \\
{$[48]$} & $\ell_{4} \succ \ell_{1} \succ \ell_{3} \succ \ell_{2} \succ \ell_{5}$ \\
[49] & $\ell_{4} \succ \ell_{1} \succ \ell_{3} \succ \ell_{2} \succ \ell_{5}$ \\
Proposed & $\ell_{4} \succ \ell_{1} \succ \ell_{3} \succ \ell_{2} \succ \ell_{5}$ \\
\hline
\end{tabular}

Our main concern to compare with the method proposed in Reference [23] because both papers dealing with GIFSS. But when we solved the case study Section 5, the order of the alternatives not remains the same, that is,

$$
\ell_{3} \succ \ell_{5} \succ \ell_{4} \succ \ell_{6} \succ \ell_{1} \succ \ell_{2} \succ \ell_{7} \text {. }
$$

The reason behind the changing of order is that, in our proposed method we put some threshold function or values initially that minimize the effect of alternatives that are not fulfil the initial criteria. 
The proposed method depends on the initial threshold function, like if we use the top-bottom threshold function then we get the order of alternatives

$$
\ell_{3} \succ \ell_{5} \succ \ell_{4} \succ \ell_{1} \succ\left\{\ell_{2}, \ell_{6}, \ell_{7}\right\} .
$$

It means it is important to select the threshold function or threshold value according to the situation required in decision making. Actually this is an advantage in a sense that our proposed method response when we change the threshold function or values because the threshold values suggested according to the decision problem situation and secondly we strengthen the attributes by assigning the weights to the attributes.

The limitations of our approach are that in Algorithm 1, the attributes categorized in two groups and the weights are more effective only when the difference between positive membership and negative membership function is maximum. In Algorithm 2, we assign different weights to the attributes but to make weights more effective, the difference between positive membership and negative membership function is high.

\section{Conclusions}

Keeping in mind the idea of decision making, in this paper, we have strengthened the director/administrator/decision maker point of view because first, he adjusts the initial conditions according to a situation like in the case study in Section 5, he makes an initial condition that the CGPA of a candidate should be greater than a particular value. Secondly, he differentiates the different attributes/criteria by assigning different weights like in the case study in Section 5, he gave the interview more weightage than the personal statement. Thirdly, we provide the criteria for obtaining weights of different attributes and the weights obtained from PIFS provided by director/administrator/decision maker.

The idea of GIFSS is very encouraging in decision-making since it considers how to capitalize an additional intuitionistic fuzzy input from the director to minimize any possible perversion in the data provided by evaluating specialists. First, Skowron and Rauszer [43] initiated the concept of discernibility matrix and extensively used in rough sets to solve attribute reduction, and the influence of it are significant and easy to understand. In this paper, we use an adjustable perspective to GIFSS and get level soft sets. Then each GIFSS can be seen as a level soft set and composed a crisp soft set, therefore, for solving decision making problems we apply $\mathcal{W} \mathcal{S D} \mathcal{M}$. In literature, GIFSS is defined and applied for decision making problems using intuitionistic fuzzy weighted averaging operators. But in our daily life decision making problems, different attributes are not of equal importance. Some are more important than others; therefore, the decision maker assigns different values (weights) to different attributes and imposed different threshold functions when we need to put a restriction on membership functions and non-membership functions. Our proposed technique can not only give the best alternative but also an order relation of all alternatives easily, by scanning the $\mathcal{W S D} \mathcal{M}$ at one time. We define the threshold functions like mid-threshold, top-bottom-threshold, bottom-bottom-threshold, top-top-threshold, med-threshold function and their level soft sets. After, we proposed two algorithms based on threshold functions, $\mathcal{W} \mathcal{S D M}$, and GIFSSs. In Algorithm 1, the attributes are categorized in two groups while in Algorithm 2, each attribute is weighted differently. To show the supremacy of the given methods we illustrate a descriptive example using Algorithm 2. Results indicate that the proposed method is more effective and generalized over all the existing methods of fuzzy soft sets.

In future directions, we will introduce the different methods to get the weights of attributes by using similarity or entropy measures. We apply this method to the best concept selection and multiattribute classification or sorting problems. 
Author Contributions: All authors contributed equally in this research paper.

Funding: Petchra Pra Jom Klao Ph.D. Research Scholarship from King Mongkut's University of Technology Thonburi (KMUTT) and Theoretical and Computational Science (TaCS) Center. Moreover, Poom Kumam was supported by the Thailand Research Fund and the King Mongkut's University of Technology Thonburi under the TRF Research Scholar Grant No. RSA6080047. The Rajamangala University of Technology Thanyaburi (RMUTTT) (Grant No. NSF62D0604).

Acknowledgments: This project was supported by Center of Excellence in Theoretical and Computational Science (TaCS-CoE), KMUTT. The first author was supported by the "Petchra Pra Jom Klao Ph.D. Research Scholarship from King Mongkut's University of Technology Thonburi". Moreover, this research work was financially supported by the Rajamangala University of Technology Thanyaburi (RMUTTT) (Grant No. NSF62D0604).

Conflicts of Interest: The authors declare no conflict of interest.

\begin{tabular}{|c|c|}
\hline \\
\hline \multicolumn{2}{|c|}{$\begin{array}{l}\text { Abbreviations } \\
\text { The following abbreviations are used in this manuscr }\end{array}$} \\
\hline IFS & Intuitionistic Fuzzy Set \\
\hline IFSS & Intuitionistic Fuzzy Soft Set \\
\hline GIFSS & Generalized Intuitionistic Fuzzy Soft Set \\
\hline$S D P$ & Soft Discernibility Parameters \\
\hline $\mathcal{S D} \mathcal{M}$ & Soft Discernibility Matrix \\
\hline $\begin{array}{l}\mathcal{W} \mathcal{S D M} \\
\text { w.r.t. }\end{array}$ & $\begin{array}{l}\text { Weighted Soft Discernibility Matrix } \\
\text { with respect to }\end{array}$ \\
\hline
\end{tabular}

\section{References}

1. Zadeh, L.A. Fuzzy sets. Inf. Control 1965, 8, 338-353. [CrossRef]

2. Gau, W.L.; Buehrer, D.J. Vague sets. IEEE Trans. Syst. Man Cybern. 1993, 23, 610-614. [CrossRef]

3. Pawlak, Z. Rough sets. Int. J. Comput. Inf. Sci. 1982, 11, 341-356. [CrossRef]

4. Atanassov, K.T. Intuitionistic fuzzy sets. Fuzzy Sets Syst. 1986, 20, 87-96. [CrossRef]

5. Gorzalzany, M.B. A method of inference in approximate reasoning based on interval-valued fuzzy sets. Fuzzy Sets Syst. 1987, 21, 1-17. [CrossRef]

6. Molodtsov, D. Soft set theory-first results. Comput. Math. Appl. 1999, 37, 19-31. [CrossRef]

7. Molodtsov, D. The Theory of Soft Sets; URSS Publishers: Moscow, Russia, 2004. (In Russian)

8. Maji, P.K.; Biswas, R.; Roy, A.R. Soft set theory. Comput. Math. Appl. 2003, 45, 555-562. [CrossRef]

9. Zou, Y.; Xiao, Z. Data analysis approaches of soft sets under incomplete information. Knowl. Based Syst. 2008, 21, 941-945. [CrossRef]

10. Xiao, Z.; Gong, K.; Zou, Y. A combined forecasting approach based on fuzzy soft sets. J. Comput. Math. Appl. 2009, 228, 326-333. [CrossRef]

11. Maji, P.K.; Biswas, R.; Roy, A.R. Fuzzy soft sets. J. Fuzzy Math. 2001, 9, 589-602.

12. Maji, P.K.; Biswas, R.; Roy, A.R. Intuitionistic fuzzy soft sets. J. Fuzzy Math. 2001, 9, 677-692.

13. Majumdar, P.; Samanta, S.K. Generalised fuzzy soft sets. Comput. Math. Appl. 2010, 59, 1425-1432. [CrossRef]

14. Yang, X.B.; Lin, T.Y.; Yang, J.Y.; Li, Y.; Yu, D.Y. Combination of interval-valued fuzzy set and soft set. Comput. Math. Appl. 2009, 58, 521-527. [CrossRef]

15. Ali, M.I. A note on soft sets, rough soft sets and fuzzy soft sets. Appl. Soft Comput. 2011, 11, 3329-3332.

16. Xu, W.; Ma, J.; Wang, S.; Hao, G. Vague soft sets and their properties. Comput. Math. Appl. 2010, 59, 787-794. [CrossRef]

17. Xiao, Z.; Xia, S.; Gong, K.; Li, D. The trapezoidal fuzzy soft set and its application in MCDM. Appl. Math. Model. 2012, 36, 5844-5855. [CrossRef]

18. Maji, P.K. Neutrosophic soft set. Ann. Fuzzy Math. Inform. 2013, 5, 57-168.

19. Broumi, S.; Smarandache, F. Intuitionistic neutrosophic soft set. J. Inf. Comput. Sci. 2013, 8, 130-140. 
20. Yang, Y.; Tan, X.; Meng, C.C. The multi-fuzzy soft set and its application in decision making. Appl. Math. Model. 2013, 37, 4915-4923. [CrossRef]

21. Wang, F.; Li, X.; Chen, X. Hesitant fuzzy soft set and its applications in multicriteria decision making. J. Appl. Math. 2014, 2014. [CrossRef]

22. Agarwal, M.; Biswas, K.K.; Hanmandlu, M. Generalized intuitionistic fuzzy soft sets with applications in decision-making. Appl. Soft Comput. 2013, 13, 3552-3566. [CrossRef]

23. Feng, F.; Fujita, H.; Ali, M.I.; Yager, R.R. Another view on generalized intuitionistic fuzzy soft sets and related multi attribute decision making methods. IEEE Trans. Fuzzy Syst. 2018, 27, 474-488. [CrossRef]

24. Cuong, B.C. Picture fuzzy sets. J. Comput. Sci. Cybern. 2014, 30, 409-420.

25. Singh, P. Correlation coefficients for picture fuzzy sets. J. Intell. Fuzzy Syst. 2014, 27, 2857-2868.

26. Thong, P.H.; Son, L.H. A new approach to multi-variables fuzzy forecasting using picture fuzzy clustering and picture fuzzy rules interpolation method. In Proceedings of the 6th International Conference on Knowledge and Systems Engineering, Hanoi, Vietnam, 9-11 January 2015; pp. 679-690.

27. Son, L.H.; Viet, P.; Hai, P. Picture inference system: A new fuzzy inference system on picture fuzzy set. Appl. Intell. 2016, 46, 652-669. [CrossRef]

28. Thong, P.H.; Son, L.H. A novel automatic picture fuzzy clustering method based on particle swarm optimization and picture composite cardinality. Knowl. Based Syst. 2016, 109, 48-60. [CrossRef]

29. Wei, G. Picture fuzzy aggregation operator and their application to multiple attribute decision making. J. Int. Fuzzy Syst. 2017, 33, 713-724. [CrossRef]

30. Wei, G.W. Picture fuzzy cross-entropy for multiple attribute decision making problems. J. Bus. Econ. Manag. 2016, 17, 491-502. [CrossRef]

31. Garg, H. Some picture fuzzy aggregation operators and their applications to multi criteria decision-making. Arab. J. Sci. Eng. 2017, 42, 5275-5290. [CrossRef]

32. Yang, Y.; Liang, C.; Ji, S.; Liu, T. Adjustable soft discernibility matrix based on picture fuzzy soft sets and its application in decision making. J. Int. Fuzzy Syst. 2015, 29, 1711-1722. [CrossRef]

33. Khan, M.J.; Kumam, P.; Ashraf, S.; Kumam, W. Generalized Picture Fuzzy Soft Sets and Their Application in Decision Support Systems. Symmetry 2019, 11, 415. [CrossRef]

34. Liu, Z.; Qin, K.; Pei, Z. A Method for Fuzzy Soft Sets in Decision-Making Based on an Ideal Solution. Symmetry 2017, 9, 246. [CrossRef]

35. Ashraf, S.; Mahmood, T.; Abdullah, S.; Khan, Q. Different approaches to multi-criteria group decision making problems for picture fuzzy environment. Bull. Braz. Math. Soc. New Ser. 2019, 50, 393-397. [CrossRef]

36. Ashraf, S.; Abdullah, S.; Qadir, A. Novel concept of cubic picture fuzzy sets. J. New Theory 2018, 24, 59-72.

37. Zeng, S.; Asharf, S.; Arif, M.; Abdullah, S. Application of Exponential Jensen Picture Fuzzy Divergence Measure in Multi-Criteria Group Decision Making. Mathematics 2019, 7, 191. [CrossRef]

38. Muhammad, Q.; Abdullah, S.; Asharf, S. Solution of multi-criteria group decision making problem based on picture linguistic informations. Int. J. Algebra Stat. 2019, 8, 1-11.

39. Cagman, N.; Enginoglu, S. Soft matrix theory and its decision making. Comput. Math. Appl. 2010, 59, 3308-3314. [CrossRef]

40. Feng, Q.; Zhou, Y. Soft discernibility matrix and its applications in decision making. Appl. Soft Comput. 2014, 24, 749-756. [CrossRef]

41. Feng, F.; Jun, Y.B.; Liu, X.; Li, L. An adjustable approach to fuzzy soft set based decision making. J. Comput. Appl. Math. 2010, 234, 10-20. [CrossRef]

42. Jiang, Y.; Tang, Y.; Chen, Q. An adjustable approach to intuitionistic fuzzy soft sets based decision making. Appl. Math. Model. 2011, 35, 824-836. [CrossRef]

43. Skowron, A.; Rauszer, C. The discernibility matrices and functions in information systems. In Intelligent Decision Support: Handbook of Applications and Advances of Rough Sets Theory; Slowinski, R., Ed.; Kluwer Academic Publisher: Dordrecht, The Netherlands, 1992; pp. 331-362.

44. Ali, M.I. Another view on reduction of parameters in soft sets. Appl. Soft Comput. 2012, 12, 1814-1821. [CrossRef] 
45. Khalil, A.M. Commentary on "Generalized intuitionistic fuzzy soft sets with applications in decision-making". Appl. Soft Comput. 2015, 37, 519-520. [CrossRef]

46. Lin, Z.G.; Xu, L.Z.; Wang, J.Y. Multi-criteria fusion decisionmaking method based on vague set. Comput. Eng. 2005, 31, 11-13.

47. Liu, H.W. Vague set methods of multi-criteria fuzzy decision making. Syst. Eng. Theory Pract. 2004, 4, 103-109.

48. Xu, Z.S. Intuitionistic fuzzy aggregation operators. IEEE Trans. Fuzzy Syst. 2007, 15, 1179-1187.

49. Ye, J. Using an improved measure function of vague sets for multicriteria fuzzy decision making. Expert Syst. Appl. 2015, 37, 4706-4709. [CrossRef]

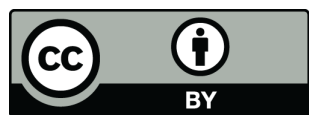

(C) 2019 by the authors. Licensee MDPI, Basel, Switzerland. This article is an open access article distributed under the terms and conditions of the Creative Commons Attribution (CC BY) license (http:/ / creativecommons.org/licenses/by/4.0/). 